\title{
Fractional glassy relaxation and convolution modules of distributions
}

\author{
T. Kleiner ${ }^{1} \cdot$ R. Hilfer ${ }^{1}$
}

Received: 30 September 2020 / Accepted: 29 January 2021 / Published online: 28 June 2021

(C) The Author(s) 2021

\begin{abstract}
Solving fractional relaxation equations requires precisely characterized domains of definition for applications of fractional differential and integral operators. Determining these domains has been a longstanding problem. Applications in physics and engineering typically require extension from domains of functions to domains of distributions. In this work convolution modules are constructed for given sets of distributions that generate distributional convolution algebras. Convolutional inversion of fractional equations leads to a broad class of multinomial Mittag-Leffler type distributions. A comprehensive asymptotic analysis of these is carried out. Combined with the module construction the asymptotic analysis yields domains of distributions, that guarantee existence and uniqueness of solutions to fractional differential equations. The mathematical results are applied to anomalous dielectric relaxation in glasses. An analytic expression for the frequency dependent dielectric susceptibility is applied to broadband spectra of glycerol. This application reveals a temperature independent and universal dynamical scaling exponent.
\end{abstract}

Keywords Convolution modules · Causal distributions · Fractional calculus · Glass transition · Excess wing

\section{Introduction}

Applications of fractional calculus in physics [3,5,9,21] and mathematics [16,40,49] are enjoying an undiminished surge of attention in recent years. Determining extended domains and parameter ranges for fractional operators is oftentimes the crucial step in advanced applications.

Modern interpretations of fractional derivatives and integrals have for many years centered around fractional powers of closed operators on Banach spaces $[2,12,15$,

R. Hilfer

hilfer@icp.uni-stuttgart.de

1 Fakultät Mathematik und Physik, Universität Stuttgart, Allmandring 3, 70569 Stuttgart, Deutschland

Birkhäuser 
17,20,47]. A paradigmatic class of closed operators for such studies are generators $H=T^{\prime}(0)$ of strongly continuous semigroups $T(s)$. It is common $[23,45,46]$ to use Marchaud's interpretation [26] of fractional derivatives rewritten as

$$
(-H)^{\alpha} f=\lim _{\varepsilon \rightarrow 0} \frac{1}{C_{\alpha, m}} \int_{\varepsilon}^{\infty} \frac{[\mathbf{1}-T(s)]^{m} f}{s^{\alpha+1}} d s,
$$

where $T(s)$ is an equibounded semigroup (taken to be translation in [26]) with infinitesimal generator $H$ on a Banach space $X, f \in X, m \in \mathbb{N}, \alpha \in \mathbb{R}, C_{\alpha, m} \in \mathbb{R}$ is a norming constant, and the limit is taken in the norm topology. Other interpretations based on rewriting Cauchy's integral formula with the help of resolvents are applicable to sectorial operators in Hilbert spaces [29]. Resolvents were also crucial in Balakrishnan's celebrated interpretation [2]. Extending a commutative ring of complex-valued continuous functions to a field of ordered pairs representing convolution quotients provides a more algebraic interpretation of fractional calculus [13,30,48]. Mellin transform based interpretations of fractional powers of operators in Banach spaces are useful for extensions to purely imaginary powers $[38,44]$

Distributional extensions of fractional calculus have received comparatively little attention, perhaps due to the fact that distribution spaces tend to be locally convex but not normable. Erdelyi [6] and McBride [28] have considered distributions on test functions with half-axis support, while the full real axis was considered in [22,41]. In [27] Balakrishnan's interpretation was extended abstractly to non-negative operators on locally convex spaces without, however, providing concrete spaces of distributions for applications.

Given the need for concretely characterized extended domains in applications a first step was taken in $[18,19]$ where fractional Weyl integrals have been interpreted as convolutions of Radon measures with continuous functions. Locally convex topologies generated by weighted supremum norms were identified such that a given set of convolution operators acts as an equicontinuous family of endomorphisms on certain weighted spaces of continuous functions [19]. Our objective in this work is to combine the benefits of our approach [18,19] with those of Marchaud's [26], with those of fractional powers $[2,12,15,17,20,47]$, with those of translation invariant distribution spaces $[22,41]$, and with the algebraic benefits of operational calculus $[13,30,48]$. Recall that in Marchaud's interpretation [26] fractional derivatives with positive orders impose rather mild growth restrictions and are well defined also on bounded functions. In $[22,41]$, on the other hand, the domains are differentiation and translation invariant distribution spaces, but with rather strong growth restrictions. Abstract operational approaches $[13,30,48]$ or fractional powers (with appropriate domain restrictions) $[2,12,15,17,20,47]$ automatically guarantee invertibility and the validity of algebraic relations between operators. Merging these benefits with the advantage of "maximal" domains for certain sets of integral operators on weighted spaces in $[18,19]$ has therefore been the objective of this work.

The result is an extended distributional translation invariant fractional calculus with the growth conditions as mild as possible while preserving composition laws. The calculus emerges naturally from a construction that associates a convolution module 
to any given convolution semigroup of distributions in such a way that the resulting module is the maximal distribution space with respect to simultaneous convolution of multiple distributions from the semigroup. Our calculus guarantees the invertibility of linear combinations of fractional derivatives on an appropriate domain that depends only on the asymptotic behaviour of the convolutional inverses at infinity. The approach is less abstract than algebraic approaches or fractional power approaches, but still has the benefits of the latter.

Results in this work are based on considering the convolution field of causal distributions $\mathscr{F}_{+}$generated by the convolution kernels of fractional integrals $\mathrm{I}_{+}^{\alpha}$ and derivatives $\mathrm{D}_{+}^{\alpha}$ which we denote as $\mathrm{p}_{+}^{\alpha}$ and $\mathrm{q}_{+}^{\alpha}$. In Sect. 3 we show using Neumann series, that the quotients from the field $\mathscr{F}_{+}$can be identified as linear combinations of a certain multiparameter family of distributions specified in Definition 2. A thorough study of their properties is carried out in Propositions 1 and 2. Multinomial MittagLeffler functions characterize their densities. In Sect. 4 an explicit analysis based on Hankel type integral representations (Theorem 2) serves to characterize the asymptotic behaviour of these distributions in Theorem 3. To the best of our knowledge this represents the most comprehensive asymptotic analysis of multinomial Mittag-Leffler distributions to date. For $t \rightarrow+\infty$ they behave asymptotically as $t^{p} \mathrm{e}^{a t}$ with certain $a, p \in \mathbb{R}$ (Theorem 4) leading to a classification into four types described in Theorem 5. The classification involves the set of singularities of the Laplace transforms given in Theorem 6 and induces four corresponding subalgebras of $\mathscr{F}_{+}$that are introduced in Definition 3.

The main results of the paper are derived from a new method to construct endomorphic domains of distributions for general families of convolution operators that is described in Sect. 6. Let $A$ be a given set of distributions with the property that $A$ generates a convolution algebra. A convolution module $(A)_{\mathscr{D}^{\prime}}^{* \mathrm{M}}$ of distributions is introduced in Definition 5 such that $A$ operates linearly and associatively on $(A)_{\mathscr{D}^{\prime}}^{* \mathrm{M}}$ as described in Theorem 8 . The distributions belonging to $(A)_{\mathscr{D}^{\prime}}^{* \mathrm{M}}$ are characterized in Theorem 9. The key for this construction is the notion of convolutes and convolvability of pairs and tuples of distributions (see Refs. [43, p. 2], [34, Definition 5]).

The module construction from Definition 3 is then applied to the subalgebras of $\mathscr{F}_{+}$in Theorem 10 of Sect. 7. Characterizations of the resulting modules are obtained from the asymptotic results in Sect. 4 and Theorem 9. Conclusions are drawn in Sect. 8 such as existence and uniqueness of solutions for translation invariant fractional linear response equations on certain spaces of distributions. Fractional derivatives are seen to be well behaved on a space of distributions $\mathscr{D}_{L^{1}\left(Q_{-}\right)}^{\prime}$ that contains all distributions that are bounded on the left half axis. In this way the index law for fractional derivatives is extended to a distribution space considerably larger than those in previous approaches, because associativity holds for the convolution module $\mathscr{D}_{L^{1}\left(Q_{-}\right)}^{\prime}$ over the algebra generated by $\mathrm{q}_{+}^{\alpha}, \alpha \in \mathbb{R}_{+}$.

The mathematical results are applied in Sect. 9 to the longstanding problem of anomalous dielectric relaxation in supercooled liquids and glass formers. The domain $\mathscr{D}_{L^{1}\left(Q_{-}\right)}^{\prime}$ is found to be well adapted for the solution of fractional relaxation problems as linear response equations, because the reverse Heaviside function $\Theta_{-}$and periodic distributions, such as $\mathrm{e}^{\mathrm{i} \omega}, \omega \in \mathbb{R}$, both belong to $\mathscr{D}_{L^{1}\left(Q_{-}\right)}^{\prime}$. This permits to view 
dielectric relaxation processes, described by fractional initial value problems on $\mathbb{R}_{+}$, and the response to periodic excitations, described by Fourier multipliers on $\mathbb{R}$, as resulting from a single translation invariant linear differential equation on $\mathbb{R}$.

\section{Notations}

The sets of natural, integer, real and complex numbers are $\mathbb{N}=\{0,1, \ldots\}, \mathbb{Z}, \mathbb{R}, \mathbb{C}$, as usual. The notations $\mathbb{C}_{\neq 0}=\mathbb{C} \backslash\{0\}, \mathbb{R}_{+}=\{x \in \mathbb{R}: x>0\}, \overline{\mathbb{R}}_{+}=[0,+\infty]$, and $\mathbb{H}:=\mathbb{H}_{0}$ are used, where $\mathbb{H}_{\sigma}:=\{z \in \mathbb{C}: \mathfrak{R} z>\sigma\}$ is a complex half plane. The symbol $\mathbb{L}$ denotes the Riemann surface of the logarithm. Closed annuli and sectors in $\mathbb{L}$ are denoted as

$$
\begin{aligned}
\mathbb{A}(r, R) & :=\{\zeta \in \mathbb{L}: r \leq|\zeta| \leq R\}, \quad \mathbb{S}(\theta):=\{\zeta \in \mathbb{L}:|\arg \zeta| \leq \theta\}, \\
\mathbb{A}(r, R ; \theta) & :=\mathbb{A}(r, R) \cap \mathbb{S}(\theta)
\end{aligned}
$$

for $0 \leq r \leq R \leq \infty$ and $\theta \in \mathbb{R}_{+}$. The Hankel loop $\mathrm{H}(r ; \theta)$ with parameters $r, \theta \in \mathbb{R}_{+}$ is the piecewise smooth path $\eta_{r ; \theta}: \mathbb{R} \rightarrow \mathbb{L}$ that encircles the domain $\mathbb{L} \backslash \mathbb{A} \mathbb{S}(r,+\infty ; \theta)$ counter clockwise with speed 1 and fulfills $\eta_{r ; \theta}(0)=r$.

The inverse exp: $\mathbb{C} \rightarrow \mathbb{L}$ of the logarithm is an injective variant of the exponential function. Any $\zeta \in \mathbb{L}$ can be written as $\zeta=r \widetilde{\mathrm{e}}^{\mathrm{i} \phi}$ with unique $r \in \mathbb{R}_{+}$and $\phi \in \mathbb{R}$. Multiplication and exponentiation in $\mathbb{L}$ are given by the formulas

$$
\begin{aligned}
\zeta \cdot \xi & =\left(r \widetilde{\mathrm{e}}^{\mathrm{i} \phi}\right) \cdot\left(s \widetilde{\mathrm{e}}^{\mathrm{i} \psi}\right)=(r \cdot s) \widetilde{\mathrm{e}}^{\mathrm{i}(\phi+\psi)} & & \text { for all } \zeta, \xi \in \mathbb{L}, \\
\zeta^{\alpha} & =r^{\Re \alpha} \mathrm{e}^{-\phi \Im \alpha} \widetilde{\exp }(\mathrm{i}(\phi \cdot \Im \alpha+\log r \cdot \Re \alpha)) & & \text { for all } \zeta \in \mathbb{L}, \alpha \in \mathbb{C},
\end{aligned}
$$

with $\Re$ and $\Im$ denoting real and imaginary parts. The exponential laws

$$
(\zeta \cdot \xi)^{\alpha}=\zeta^{\alpha} \cdot \xi^{\alpha}, \quad \zeta^{\alpha} \cdot \zeta^{\beta}=\zeta^{\alpha+\beta}, \quad\left(\zeta^{\alpha}\right)^{\beta}=\zeta^{\alpha \cdot \beta}
$$

hold for all $\zeta, \xi \in \mathbb{L}, \alpha, \beta \in \mathbb{C}$. The canonical projection $\mathbb{L} \rightarrow \mathbb{C}_{\neq 0} \subseteq \mathbb{C}, r \widetilde{\mathrm{e}}^{\mathrm{i} \phi} \mapsto$ $r \mathrm{e}^{\mathrm{i} \phi}$, corresponds to the quotient mapping $\phi \mapsto \phi+2 \pi \mathbb{Z}$. Numbers $\zeta \in \mathbb{L}$ occuring as summands, or multiplied by a number $z \in \mathbb{C}$, are implicitly converted to numbers from $\mathbb{C}$. Real part, imaginary part and absolute value are defined similarly, but the argument of $\zeta=r \widetilde{\mathrm{e}}^{\mathrm{i} \phi} \in \mathbb{L}$ is $\arg \zeta=\phi$. With these conventions, the functions

$$
\zeta \mapsto \frac{\Sigma \lambda \zeta^{\alpha}}{\Sigma \mu \zeta^{\beta}}=\frac{\lambda_{1} \zeta^{\alpha_{1}}+\cdots+\lambda_{n} \zeta^{\alpha_{n}}}{\mu_{1} \zeta^{\beta_{1}}+\cdots+\mu_{m} \zeta^{\beta_{m}}}
$$

are well-defined as meromorphic functions on $\mathbb{L}$ for parameters $n, m \in \mathbb{N}, \alpha, \lambda \in \mathbb{C}^{n}$, $\beta, \mu \in \mathbb{C}^{m}$ with $m \neq 0$ and $\mu_{1}, \ldots, \mu_{m} \neq 0$. Here, if $x$ is a scalar, $y=\left(y_{1}, \ldots, y_{n}\right)$ and $z=\left(z_{1}, \ldots, z_{n}\right)(n \in \mathbb{N})$, then the shorthand notations $x y=\left(x y_{1}, \ldots, x y_{n}\right)$, $x^{y}=\left(x^{y_{1}}, \ldots, x^{y_{n}}\right), y z=\left(y_{1} z_{1}, \ldots, y_{n} z_{n}\right), y^{z}=\left(y_{1}^{z_{1}}, \ldots, y_{n}^{z_{n}}\right), \Sigma y:=y_{1}+$ $\cdots+y_{n}$ and $\Pi y:=y_{1} \cdots y_{n}$ are used for products, powers and sums. Multinomial coefficients are denoted as 


$$
[[p]]:=\frac{(\Sigma p) !}{\Pi p !}=\frac{\left(p_{1}+\cdots+p_{n}\right) !}{p_{1} ! \cdots p_{n} !}
$$

for all $p \in \mathbb{N}^{n}$. In our notation the multinomial formula reads as

$$
[\Sigma \lambda]^{q}=\sum_{q=\Sigma p}[[p]] \cdot \Pi\left(\lambda^{p}\right)
$$

with sum range $p \in \mathbb{N}^{n}$ for all $\lambda \in \mathbb{C}^{n}$ and $q \in \mathbb{N}$.

Except for Sect. 7 all function spaces consist of functions on $\mathbb{R}$. The function spaces of interest are

$$
\begin{aligned}
L_{\text {loc }}^{1} & :=\{f: \mathbb{R} \rightarrow \mathbb{C} \mid f \text { is locally integrable }\} \\
\mathscr{C}^{m} & :=\{f: \mathbb{R} \rightarrow \mathbb{C} \mid f \text { is } m \text {-times continuously differentiable }\} \\
\mathscr{D} & :=\left\{f \in \mathscr{C}^{\infty} \mid f \text { has compact support }\right\} \\
\mathscr{S} & :=\left\{f \in \mathscr{C}^{\infty} \mid f \text { and its derivatives decay rapidly at } \infty\right\} \\
\mathscr{B} & :=\left\{f \in \mathscr{C}^{\infty} \mid f \text { has bounded derivatives }\right\} \\
\mathscr{I} & :=\left\{f: \mathbb{R} \rightarrow \overline{\mathbb{R}}_{+} \mid f \text { is lower semicontinuous }\right\}
\end{aligned}
$$

and the space of distributions is denoted $\mathscr{D}^{\prime}$. The compactly supported, tempered and integrable distributions are denoted by $\mathscr{E}^{\prime}, \mathscr{S}^{\prime}$ and $\mathscr{D}_{L^{1}}^{\prime}[36,42]$. A subscript "+", as in $\mathscr{I}_{+}, L_{\text {loc, }+}^{1}, \mathscr{C}_{+}^{m}$ and $\mathscr{D}_{+}^{\prime}$, indicates spaces of functions or distributions with support bounded on the left. The space of convolutors and multipliers is denoted by $\mathscr{O}_{C}^{\prime}$, and $\mathscr{O}_{M}[14,42]$. The space $\mathscr{O}_{C}^{\prime}$ is characterized as the set of distributions such that $\phi * \mathscr{O}_{C}^{\prime} \in \mathscr{S}$ for all test functions $\phi$. Topological statements for locally convex spaces will always refer to the usual strong topologies.

Inverses with respect to convolution in $\left(\mathscr{D}_{+}^{\prime},+, *\right)$ will be denoted as $(-)^{*-1}$. The notion of fractions will be extended to "convolution quotients" $u / v=u *(v)^{*-1}$ for $u, v \in \mathscr{D}_{+}^{\prime}$ whenever $v$ invertible and the meaning is clear from the context.

\section{The field generated by causal power distributions}

Definition 1 The causal power distribution $\mathrm{p}_{+}^{\alpha}$ of index $\alpha \in \mathbb{C}$ is given by

$$
\begin{aligned}
\mathrm{p}_{+}^{\alpha}(t): & =\frac{t_{+}^{\alpha-1}}{\Gamma(\alpha)}=\left\{\begin{array}{ll}
t^{\alpha-1} / \Gamma(\alpha) & \text { for } t>0, \\
0 & \text { for } t \leq 0,
\end{array} \text { for } \Re \alpha>0,\right. \\
\mathrm{p}_{+}^{\alpha}: & =\mathrm{D}^{m} \mathrm{p}_{+}^{\alpha+m}
\end{aligned}
$$


where $\mathrm{D}^{m}$ denotes the $m$-th distibutional derivative [7,36]. The inverse of $\mathrm{p}_{+}^{\alpha}$ with respect to convolution is denoted as $\mathrm{q}_{+}^{\alpha}:=\left(\mathrm{p}_{+}^{\alpha}\right)^{*-1}$. Further, define the sets

$$
\begin{array}{ll}
\mathscr{P}_{+}:=\left\{\mathrm{p}_{+}^{\alpha}: \alpha \in \mathbb{R}\right\}, & \mathscr{Q}_{+}:=\left\{\mathrm{q}_{+}^{\alpha}: \alpha \in \mathbb{R}_{+}\right\}, \\
\mathscr{P}_{+}^{\mathbb{C}}:=\left\{\mathrm{p}_{+}^{\alpha}: \alpha \in \mathbb{C}\right\}, & \mathscr{Q}_{+}^{\mathbb{H}}:=\left\{\mathrm{q}_{+}^{\alpha}: \alpha \in \mathbb{H}\right\} .
\end{array}
$$

The goal of this section is to prove that the convolution subgroup $\mathscr{P}_{+}$of $\mathscr{D}_{+}^{\prime}$, that consists of the causal power distributions with real indices, generates a field $\mathscr{F}_{+}=\left(\mathbb{C}\left(\mathscr{P}_{+}\right),+, *\right)$. This is achieved using the Neumann series method to calculate the inverses of $\mathbb{C}\left[\mathscr{P}_{+}\right] \backslash\{0\}$ within $\left(\mathscr{D}_{+}^{\prime},+, *\right)$. Representations for these inverses are given in terms of generalized multivariate Mittag-Leffler functions. The inverses are shown to be analytic in indices and prefactors.

A causal power distribution fulfills supp $\mathrm{p}_{+}^{\alpha}=\{0\}$ if and only if $\alpha \in-\mathbb{N}$, and then $\mathrm{p}_{+}^{-m}=\mathrm{D}^{m} \delta$ where $\delta$ is the Dirac-distribution. Otherwise supp $\mathrm{p}_{+}^{\alpha}=[0,+\infty)$. For example, $p_{+}^{1}=\Theta_{+}$is just the Heaviside function. The index law holds, which reads

$$
\mathrm{p}_{+}^{\alpha} * \mathrm{p}_{+}^{\beta}=\mathrm{p}_{+}^{\alpha+\beta} \quad \text { for all } \alpha, \beta \in \mathbb{C}
$$

and entails that $\mathrm{q}_{+}^{\alpha}=\left(\mathrm{p}_{+}^{\alpha}\right)^{*-1}=\mathrm{p}_{+}^{-\alpha}$. As a conclusion, $\mathscr{P}_{+}$and $\mathscr{P} \mathbb{C}$ are convolution groups and $\mathscr{Q}_{+}$and $\mathscr{Q}_{+}^{\mathbb{H}}$ are convolution semigroups contained in $\mathscr{D}_{+}^{\prime}$. The subgroup $\left\{\mathrm{p}_{+}^{k}: k \in \mathbb{Z}\right\} \subseteq \mathscr{P}_{+}$is just the group of differentiation and formation of the unique primitive with support bounded on the left.

The mappings $\mathrm{I}_{+}^{\alpha}: u \mapsto \mathrm{p}_{+}^{\alpha} * u$ and $\mathrm{D}_{+}^{\alpha}: u \mapsto \mathrm{q}_{+}^{\alpha} * u$ are understood as fractional integration and differentiation operators. Due to the fact that $\left(\mathscr{D}_{+}^{\prime},+, *\right)$ is a commutative, associative and hypo-continuous convolution algebra, see [42, Ch. VI, §5], I ${ }_{+}^{\alpha}$ and $\mathrm{D}_{+}^{\alpha}$ are well-defined continuous linear operators $\mathscr{D}_{+}^{\prime} \rightarrow \mathscr{D}_{+}^{\prime}$ that are bijective and fulfill the index laws $\mathrm{I}_{+}^{\alpha} \circ \mathrm{I}_{+}^{\beta}=\mathrm{I}_{+}^{\alpha+\beta}$ and $\mathrm{D}_{+}^{\alpha} \circ \mathrm{D}_{+}^{\beta}=\mathrm{D}_{+}^{\alpha+\beta}$ for all $\alpha, \beta \in \mathbb{C}$. These are the essentials of Schwartz' approach to fractional calculus [42].

The convolution algebra $\left(\mathbb{C}\left[\mathscr{P}_{+}^{\mathbb{C}}\right],+, *\right)$ of all linear combinations of causal power distributions is now considered as a subalgebra of $\left(\mathscr{D}_{+}^{\prime},+, *\right)$. It natural to ask which of the distributions $\Sigma \lambda \mathrm{p}_{+}^{\alpha} \neq 0$ are invertible as elements of $\left(\mathscr{D}_{+}^{\prime},+, *\right)$ which has no zero divisors [42]. This is an open problem for general indices $\alpha \in \mathbb{C}^{n}$. Using the Neumann series method, as in [33], the inverses of $\mathrm{p}_{+}^{-\gamma} *\left(\delta-\Sigma \lambda \mathrm{p}_{+}^{\alpha}\right)$ are calculated in the following when $(\alpha, \gamma, \lambda) \in \mathbb{H}^{n} \times \mathbb{C} \times \mathbb{C}^{n}, n \in \mathbb{N}$. Strong analyticity in the parameters $(\alpha, \gamma, \lambda)$ is proved, which supplements previous investigations.

Definition 2 For $n \in \mathbb{N},(\alpha, \gamma, \lambda) \in \mathbb{H}^{n} \times \mathbb{C} \times \mathbb{C}^{n}$ define the distribution

$$
\mathrm{F}_{\lambda ;+}^{\alpha ; \gamma}:=\sum_{p \in \mathbb{N}^{n}}[[p]] \cdot \Pi\left(\lambda^{p}\right) \cdot \mathrm{p}_{+}^{\gamma+\Sigma \alpha p}
$$

It is intended that $\mathrm{F}_{\emptyset ;+}^{\emptyset ; \gamma}=\mathrm{p}_{+}^{\gamma}$ when $n=0$ and one denotes $\mathrm{F}_{\lambda ;+}^{\alpha}:=\mathrm{F}_{\lambda ;+}^{\alpha ; \gamma}$.

The restriction of the distribution $\mathrm{p}_{+}^{\alpha}, \alpha \in \mathbb{C}$ to $(0,+\infty)$ has an analytic density that extends to $\mathbb{L}$ by the formula $\xi \mapsto \mathrm{p}_{+}^{\alpha}(\xi)=\xi^{\alpha-1} / \Gamma(\alpha)$. Considering (11) as a 
series of analytic functions on $\mathbb{L}$ leads to the multinomial Mittag-Leffler function of index $(\alpha, \gamma)[13,24$, eq. (29)] which is defined by the power series

$$
\mathrm{E}_{\alpha ; \gamma}(z):=\sum_{p \in \mathbb{N}^{n}} \frac{[[p]] \cdot \Pi\left(z^{p}\right)}{\Gamma(\gamma+\Sigma \alpha p)} \quad \text { for all } z \in \mathbb{C}^{n},
$$

and defines an entire function.

Proposition 1 Let $n \in \mathbb{N}, K \subseteq \mathbb{H}^{n} \times \mathbb{C} \times \mathbb{C}^{n}$ compact, $\phi \in \mathbb{R}$ and $r, R, \theta \in \mathbb{R}_{+}$ with $r \leq R$. The restriction of $\mathrm{F}_{\lambda ;+}^{\alpha ; \gamma}$ to $(0,+\infty)$ has a Lebesgue density $\mathrm{F}_{\lambda ;+}^{\alpha ; \gamma}(t)$, $t \in(0,+\infty)$. The density $\mathrm{F}_{\lambda ;+}^{\alpha ; \gamma}$ has an analytic extension to $\mathbb{L}$ that is described by the formulas

$$
\begin{aligned}
& \mathrm{F}_{\lambda ;+}^{\alpha ; \gamma}(\xi)=\sum_{p \in \mathbb{N}^{n}}[[p]] \cdot \Pi\left(\lambda^{p}\right) \cdot \mathrm{p}_{+}^{\gamma+\Sigma \alpha p}(\xi), \\
& \mathrm{F}_{\lambda ;+}^{\alpha ; \gamma}(\xi)=\xi^{\gamma-1} \cdot \mathrm{E}_{\alpha ; \gamma}\left(\lambda \xi^{\alpha}\right) .
\end{aligned}
$$

The series (13a) converges uniformly in $(\xi, \alpha, \gamma, \lambda) \in \mathbb{A} \mathbb{S}(r, R ; \theta) \times K$. Up to a finite number of summands the series convergences uniformly even on $\mathbb{A} \mathbb{S}(0, R ; \theta) \times K$.

Proof Formally, the equations (13) are obtained by replacing $z$ with $\lambda \xi^{\alpha} \in \mathbb{L}^{n}$. In order to prove the uniform convergence statements one uses that

$$
\left|\xi^{\alpha}\right| \leq \mathrm{e}^{\theta \max |\Im \alpha|} R^{\max \Re \alpha} \quad \text { for all } \xi \in \mathbb{A} \mathbb{S}(0, R ; \theta), R, \theta \in \mathbb{R}_{+}, R \geq 1
$$

and that for any $R^{\prime} \in \mathbb{R}_{+}, \theta^{\prime} \in[0, \pi / 2)$ there exist $a, C \in \mathbb{R}_{+}$such that

$$
\sup \left\{1 /|\Gamma(z)|: z \in \mathbb{A} \mathbb{S}\left(0, R^{\prime \prime} ; \theta^{\prime}\right)\right\} \leq C \exp \left(a R^{\prime \prime}\right) / \Gamma\left(R^{\prime \prime}\right) \quad \text { for all } R^{\prime \prime} \geq R^{\prime}
$$

With these inequalities the statements on convergence are seen to be consequences of the fact that $\mathrm{E}_{\alpha ; \gamma}$ is entire and that $K$ is compact.

The mapping $\mathbb{L} \times \mathbb{C} \rightarrow \mathbb{C}:(\xi, \alpha) \mapsto \mathrm{p}_{+}^{\alpha}(\xi)$ is analytic. Further, the assignment $\alpha \mapsto \mathrm{q}_{+}^{\alpha}$ is analytic as a mapping $\mathbb{C} \rightarrow \mathscr{D}_{+}^{\prime}, \mathbb{H} \rightarrow L_{\text {loc, }+}^{1}$ and $\mathbb{H}_{m+1} \rightarrow \mathscr{C}_{+}^{m}$, $m \in \mathbb{N}$. Similarly, for the functions $\mathrm{F}_{\lambda ;+}^{\alpha ; \gamma}$, one obtains the following Proposition. For Definitions of analyticity see [36] and referencies therein. For absolute convergence in locally convex spaces see [37].

Proposition 2 The series (11) converges absolutely in $\mathscr{D}_{+}^{\prime}$. The convergence is uniform in the parameters $(\alpha, \gamma, \lambda) \in K$ for any compact $K \subseteq \mathbb{H}^{n} \times \mathbb{C} \times \mathbb{C}^{n}, n \in \mathbb{N}$.

If $\Re \gamma>0$, then $\mathrm{F}_{\lambda ;+}^{\alpha ; \gamma}$ has a Lebesgue density and (11) converges absolutely and uniformly in $L_{\mathrm{loc},+}^{1}$ for $(\alpha, \gamma, \lambda) \in K$ with $K \subseteq \mathbb{H}^{n} \times \mathbb{H} \times \mathbb{C}^{n}$ compact.

If $\Re \gamma>m+1, m \in \mathbb{N}$ then $\mathrm{F}_{\lambda ;+}^{\alpha ; \gamma}$ has a density that is $m$-times continuously differentiable and (11) converges absolutely and uniformly in $\mathscr{C}_{+}^{m}$ for $(\alpha, \gamma, \lambda) \in K$ with $K \subseteq \mathbb{H}^{n} \times \mathbb{H}_{m+1} \times \mathbb{C}^{n}$ compact. 
The assignment $(\alpha, \gamma, \lambda) \mapsto \mathrm{F}_{\lambda ;+}^{\alpha ; \gamma}$ is analytic as a mapping $\mathbb{H}^{n} \times \mathbb{C} \times \mathbb{C}^{n} \rightarrow \mathscr{D}_{+}^{\prime}$, $\mathbb{H}^{n} \times \mathbb{H} \times \mathbb{C}^{n} \rightarrow L_{\text {loc },+}^{1}$ or $\mathbb{H}^{n} \times \mathbb{H}_{m+1} \times \mathbb{C}^{n} \rightarrow \mathscr{C}_{+}^{m}$

Proof The main argument of the proof is that series of analytic functions that converge absolutely on compact sets produce analytic functions and that continous linear mappings preserve absolute convergence. It holds that $\mathrm{D}^{m} \mathrm{p}_{+}^{\beta}=\mathrm{p}_{+}^{\beta-m}$ in the $\mathscr{C}^{m}$-sense whenever $m \in \mathbb{N}$ and $\beta \in \mathbb{H}_{m+1}$. Therefore, an application of Theorem 1 yields absolute and uniform convergence of (11) in $\mathscr{C}_{+}^{m}$ for $K \subseteq \mathbb{H}^{n} \times \mathbb{H}_{m+1} \times \mathbb{C}^{n}$ and thus analyticity of $(\alpha, \gamma, \lambda) \mapsto \mathrm{F}_{\lambda ;+}^{\alpha ; \gamma}$ as a mapping $\mathbb{H}^{n} \times \mathbb{H}_{m+1} \times \mathbb{C}^{n} \rightarrow \mathscr{C}_{+}^{m}$.

The canonical inclusions $\mathscr{C}_{+} \rightarrow L_{\text {loc },+}^{1} \rightarrow \mathscr{D}_{+}^{\prime}$ are continuous and the distributional derivative is a continuous linear operation on $\mathscr{D}_{+}^{\prime}$ that coincides with the classical derivative for $\mathscr{C}^{m}$-functions. Therefore, the last statement of Theorem 1 implies the analogous statements for $K \subseteq \mathbb{H}^{n} \times \mathbb{C} \times \mathbb{C}^{n}$ and $\mathscr{D}_{+}^{\prime}$ instead of $\mathscr{C}_{+}^{m}$. Finally, using that $\mathrm{p}_{+}^{\beta} \in L_{\text {loc, }+}^{1}$ whenever $\Re \beta>0$ one obtains the analogous statements for $L_{\text {loc, }+}^{1}$ when $K \subseteq \mathbb{H}^{n} \times \mathbb{H} \times \mathbb{C}^{n}$.

Proposition 3 Let $n \in \mathbb{N}$ and $(\alpha, \gamma, \lambda) \in \mathbb{H}^{n} \times \mathbb{C} \times \mathbb{C}^{n}$. It holds that

$$
\mathrm{F}_{\lambda ;+}^{\alpha ; \gamma}=\mathrm{p}_{+}^{\gamma} *\left(\delta-\Sigma \lambda \mathrm{p}_{+}^{\alpha}\right)^{*-1}=\frac{\mathrm{p}_{+}^{\gamma}}{\delta-\Sigma \lambda \mathrm{p}_{+}^{\alpha}}
$$

Proof Using (6) and (10) in the absolutely convergent series (11) one obtains

$$
\mathrm{F}_{\lambda ;+}^{\alpha ; \gamma}=\mathrm{p}_{+}^{\gamma} * \sum_{p=0}^{\infty}\left[\Sigma \lambda \mathrm{p}_{+}^{\alpha}\right]^{* p}
$$

Now the right hand side is recognized as a Neumann type series.

As a consequence of this Proposition "convolution quotients" $\left(\Sigma \lambda \mathrm{p}_{+}^{\alpha}\right) /\left(\Sigma \mu \mathrm{p}_{+}^{\beta}\right)$ with $\alpha \in \mathbb{R}^{n}, \beta_{1}<\cdots<\beta_{m}, \lambda \in \mathbb{C}^{n}, \mu \in \mathbb{C}^{m}, n, m \in \mathbb{N}$ such that $\mu_{1} \neq 0$ are well defined and can be rewritten as

$$
\frac{\Sigma \lambda \mathrm{p}_{+}^{\alpha}}{\Sigma \mu \mathrm{p}_{+}^{\beta}}=\frac{\Sigma \eta \mathrm{p}_{+}^{\gamma}}{\delta-\Sigma \kappa \mathrm{p}_{+}^{\delta}}=\sum_{k=1}^{n} \eta_{k} \mathrm{~F}_{\kappa ;+}^{\delta ; \gamma_{k}}
$$

where $\gamma_{k}:=\alpha_{k}-\beta_{1}, \eta_{k}:=\lambda_{k} / \mu_{1}, k=1, \ldots, n, \delta_{l}:=\beta_{l+1}-\beta_{1}, \kappa_{l}:=-\mu_{l+1} / \mu_{1}$, $l=1, \ldots, m-1$. This proves the main result of this section:

Theorem 1 The set of causal power distributions with real indices $\mathscr{P}_{+}$generates the field $\mathscr{F}_{+}:=\left(\mathbb{C}\left(\mathscr{P}_{+}\right),+, *\right)$ which consists of all quotients of the form $(16)$. The field $\mathscr{F}_{+}$is isomorphic to the quotient field of the ring $\mathbb{C}\left[\mathscr{P}_{+}\right]$.

\footnotetext{
${ }^{1}$ More precisely, $\mathscr{F}_{+}$is the smallest subalgebra $\mathscr{A}$ of $\left(\mathscr{D}_{+}^{\prime},+, *\right)$ such that $(\mathscr{A} \backslash\{0\})^{*-1}$ is well-defined and contained in $\mathscr{A}$ and such that $\mathscr{P}_{+} \subseteq \mathscr{A}$.
} 


\section{Asymptotic expansions of convolution quotients}

The asymptotics of the function $\mathrm{F}_{\lambda ;+}^{\alpha ; \gamma}(t)$ for $t \searrow 0$ can be conveniently described in terms of $(\alpha, \gamma, \lambda)$ using the power series representation (13a). The asymptotics for $t \rightarrow+\infty$ can be derived from the Hankel type loop integral representation under a mild restriction on the parameters $(\alpha, \gamma, \lambda)$ and are more conveniently expressed in terms of dual parameters. A tuple $(\alpha, \gamma, \lambda) \in \mathbb{H}^{n} \times \mathbb{C} \times \mathbb{C}^{n}$ is said to possess a dominant order index if there exists $l \in\{1, \ldots, n\}$ such that $\Re \alpha_{l}>\Re \alpha_{k}$ for $k=1, \ldots, n, k \neq l$ and $\mu_{l} \neq 0$. Without restrictions it can and will be assumed that $l=n$. For real $\alpha$ the assumptions $0<\alpha_{1}<\cdots<\alpha_{n}, \lambda_{n} \neq 0$ guarantee that $(\alpha, \gamma, \lambda)$ has a dominant order index.

The dual parameters $(\alpha, \gamma, \lambda) \in \mathbb{H}^{n} \times \mathbb{C} \times \mathbb{C}^{n}$ associated to any $(\beta, \delta, \mu) \in$ $\mathbb{H}^{n} \times \mathbb{C} \times \mathbb{C}^{n}$ with dominant order index are defined as

$$
\begin{array}{ll}
\alpha_{n}:=\beta_{n}, & \alpha_{k}:=\beta_{n}-\beta_{n-k} \text { for } k \neq n, \\
\lambda_{n}:=1 / \mu_{n}, & \lambda_{k}:=-\mu_{n-k} / \mu_{n} \text { for } k \neq n .
\end{array}
$$

With these parameters the distribution $\mathrm{G}_{\mu ;+}^{\beta ; \delta}$ is defined as

$$
\mathrm{G}_{\mu ;+}^{\beta ; \delta}:=\frac{\mathrm{q}_{+}^{\delta}}{\delta-\Sigma \mu \mathrm{q}_{+}^{\beta}}=-\lambda_{n} \cdot \mathrm{F}_{\lambda ;+}^{\alpha ; \gamma}
$$

Hankels' loop integral formula for the reciprocal of the Gamma function can be transformed, by means of the substitution $\zeta \mapsto \xi \zeta$, into the elegant formula

$$
\mathrm{q}_{+}^{\alpha}(\xi)=\frac{1}{2 \pi \mathrm{i}} \int_{\mathrm{H}(r ; \theta)} \mathrm{e}^{\xi \zeta} \zeta^{\alpha} \mathrm{d} \zeta \quad \text { for all } \alpha \in \mathbb{C}, \xi \in \mathbb{S}\left(\theta^{\prime}\right)
$$

where $r \in \mathbb{R}_{+}$and the angle $\theta^{\prime} \in \mathbb{R}_{+}$satisfies the condition

$$
\theta^{\prime}<\min \{\theta-\pi / 2,3 \pi / 2-\theta\}
$$

Theorem 2 Let $n \in \mathbb{N},(\beta, \delta, \mu) \in \mathbb{H}^{n} \times \mathbb{C} \times \mathbb{C}^{n}$ with dominant order index, $(\alpha, \gamma, \lambda)$ the dual parameters to $(\beta, \delta, \mu)$ and $\theta \in(\pi / 2,3 \pi / 2)$. Let $r, \theta^{\prime} \in \mathbb{R}_{+}$satisfy (20) and

$$
r>\max \{1, \sqrt[\min \Re \alpha]{\Sigma|\lambda| \exp (\max |\Im \alpha| \theta)}\}
$$

The function $\mathrm{G}_{\mu ;+}^{\beta ; \delta}(\xi)$ is described by the contour integral

$$
\mathrm{G}_{\mu ;+}^{\beta ; \delta}(\xi)=\frac{1}{2 \pi \mathrm{i}} \int_{\mathrm{H}(r ; \theta)} \frac{\zeta^{\delta}}{1-\Sigma \mu \zeta^{\beta}} \mathrm{e}^{\xi \zeta} \mathrm{d} \zeta \quad \text { for all } \xi \in \mathbb{S}\left(\theta^{\prime}\right)
$$


Proof Equation (19) is inserted into (13a). The condition (21) guarantees that exchanging the order of summation and integration is permitted because it implies that $\left|\mu \zeta^{\beta}\right|<1$ if $|\zeta| \geq r$. Recognizing a geometric series as in (15) one arrives at

$$
\mathrm{F}_{\lambda ;+}^{\alpha ; \gamma}(\xi)=\frac{1}{2 \pi \mathrm{i}} \int_{\mathrm{H}(r ; \theta)} \frac{\mathrm{e}^{\xi \zeta}}{\zeta^{\gamma}\left(1-\Sigma \lambda \zeta^{-\alpha}\right)} \mathrm{d} \zeta \quad \text { for all } \xi \in \mathbb{S}\left(\theta^{\prime}\right)
$$

Inserting here the dual parameters according to Eq. (17) yields (22).

In the following, the roots of $1-\Sigma \mu \zeta^{\beta}$ are denoted as

$$
Z_{\mu}^{\beta}:=\left\{\zeta_{0} \in \mathbb{L}: 1-\Sigma \mu \zeta_{0}^{\beta}=0\right\} \quad \text { for all }(\beta, \mu) \in \mathbb{H}^{n} \times \mathbb{C}^{n}, n \in \mathbb{N}_{1}
$$

For $\zeta_{0} \in \mathbb{L}, m_{\mu}^{\beta}\left(\zeta_{0}\right)$ will denote the greatest $m \in \mathbb{N}$ with the property that

$$
\left.\frac{\mathrm{d}^{k}}{\mathrm{~d} \zeta^{k}}\left(1-\Sigma \mu \zeta^{\beta}\right)\right|_{\zeta=\zeta_{0}=0} \quad \text { for all } k \in \mathbb{N} \text { with } k<m
$$

that means $m_{\mu}^{\beta}\left(\zeta_{0}\right)$ is the multiplicity of the root $\zeta_{0}$ of $1-\Sigma \mu \zeta^{\beta}$.

Theorem 3 Let $n,(\beta, \delta, \mu)$ and $r, \theta, \theta^{\prime}$ be as in Theorem 2. In addition, let $p \in \mathbb{R}$ and assume $\theta \notin \arg \mathrm{Z}_{\mu}^{\beta}$. The function $\mathrm{G}_{\mu ;+}^{\beta ; \delta}$ can be decomposed as

$$
\mathrm{G}_{\mu ;+}^{\beta ; \delta}(\xi)=\mathrm{E}_{\theta} \mathrm{G}_{\mu ;+}^{\beta ; \delta}(\xi)+\mathrm{P}^{p} \mathrm{G}_{\mu ;+}^{\beta ; \delta}(\xi)+\mathrm{R}_{\theta}^{p} \mathrm{G}_{\mu ;+}^{\beta ; \delta}(\xi) \quad \text { for } \xi \in \mathbb{S}\left(\theta^{\prime}\right)
$$

The $\theta$-exponential part $\mathrm{E}_{\theta} \mathrm{G}_{\mu ;+}^{\beta ; \delta}$ with $\theta \in \mathbb{R}_{+}$is defined as

$$
\mathrm{E}_{\theta} \mathrm{G}_{\mu ;+}^{\beta ; \delta}(\xi):=\sum_{\substack{\zeta_{0} \in Z_{\mu}^{\beta}:\left|\arg \zeta_{0}\right| \leq \theta \\ k \in \mathbb{N}_{1}: k \leq m_{\mu}^{\beta}\left(\zeta_{0}\right)}} \operatorname{Res}_{\zeta \rightarrow \zeta_{0}} \frac{\left(\zeta-\zeta_{0}\right)^{k-1} \zeta^{\delta}}{1-\Sigma \mu \zeta^{\beta}} \cdot \mathrm{p}_{+}^{k}(\xi) \cdot \mathrm{e}^{\xi \zeta_{0}} \quad \text { for } \xi \in \mathbb{L} .
$$

The p-negative-power part $\mathrm{P}^{p} \mathrm{G}_{\mu ;+}^{\beta ; \delta}$ with $p \in \mathbb{R}$ is defined as

$$
\mathrm{P}^{p} \mathrm{G}_{\mu ;+}^{\beta ; \delta}(\xi):=\sum_{\substack{q \in \mathbb{N}^{n} \\ \Re(\delta+\Sigma \beta q) \leq p}}[[q]] \cdot \Pi\left(\mu^{q}\right) \cdot \mathrm{q}_{+}^{\delta+\Sigma \beta q}(\xi) \quad \text { for } \xi \in \mathbb{L}
$$


The $(p, \theta)$-residual part $\mathrm{R}_{\theta}^{p} \mathrm{G}_{\mu ;+}^{\beta ; \delta}$ with $(p, \theta) \in \mathbb{R} \times(\pi / 2,3 \pi / 2) \backslash \arg \mathrm{Z}_{\mu}^{\beta}$ is

$$
\begin{array}{rlr}
\mathrm{R}_{\theta}^{p} \mathrm{G}_{\mu ;+}^{\beta ; \delta}(\xi) & :=\frac{1}{2 \pi \mathrm{i}} \lim _{r \searrow 0} \int_{\mathrm{H}(r ; \theta)} \mathrm{e}^{\xi \zeta} \zeta^{\delta} \rho_{\mu ; p}^{\beta ; \delta}(\zeta) \mathrm{d} \zeta & \text { for } \xi \in \mathbb{S}\left(\theta^{\prime}\right) . \\
\rho_{\mu ; p}^{\beta ; \delta}(\zeta) & :=\frac{1}{1-\Sigma \mu \zeta^{\beta}}-\sum_{\substack{q \in \mathbb{N}^{n} \\
\Re(\delta+\Sigma \beta q) \leq p}}[[q]] \cdot \Pi\left(\mu^{q}\right) \zeta^{\Sigma \beta q} & \text { for } \zeta \in \mathbb{L} .
\end{array}
$$

Given $r^{\prime} \in \mathbb{R}_{+}$one finds $C<\infty$ such that the $(p, \theta)$-residual part satisfies

$$
\left|\mathrm{R}_{\theta}^{p} \mathrm{G}_{\mu ;+}^{\beta ; \delta}(\xi)\right| \leq C|\xi|^{-p-1} \quad \text { for all } \xi \in \mathbb{A} \mathbb{S}\left(r^{\prime},+\infty ; \theta^{\prime}\right)
$$

Proof The function $\left(1-\Sigma \mu \zeta^{\beta}\right)^{-1}$ can be expanded in complex powers of $\zeta$ in two ways:

$$
\begin{aligned}
\frac{1}{1-\Sigma \mu \zeta^{\beta}} & =\sum_{\Re(\delta+\Sigma q \beta) \leq p}[[q]] \cdot \Pi\left(\mu^{q}\right) \cdot \zeta^{\Sigma q \beta}+\rho_{\mu ; p}^{\beta ; \delta}(\zeta) \\
& =\sum_{k \leq m-1}\left[\Sigma \mu \zeta^{\beta}\right]^{k}+\widetilde{\rho}_{\mu ; m}^{\beta}(\zeta)
\end{aligned}
$$

where $\widetilde{\rho}_{\mu ; m}^{\beta}(\zeta):=\left[\Sigma \mu \zeta^{\beta}\right]^{m} /\left(1-\Sigma \mu \zeta^{\beta}\right)$ for $m \in \mathbb{N}$. Inserting (31a) into (22) decomposes the function $\mathrm{G}_{\mu ;+}^{\beta ; \delta}(\xi)$ into the $p$-negative-power part (28) and the loop integral of $\mathrm{e}^{\xi \zeta} \zeta^{\delta} \rho_{\mu ; p}^{\beta ; \delta}(\zeta)$ over $\zeta \in \mathrm{H}(r ; \theta)$. The limit for $r \searrow 0$ of the latter is well defined. Its value is constant with the exception of a finite number of jumps, because the function $1-\Sigma \mu \zeta^{\beta}$ has only a finite number of zeros in the sector $\mathbb{S}\left(\theta^{\prime \prime}\right)$ for every $\theta^{\prime \prime} \in \mathbb{R}_{+}$. The singularities that are crossed by the loop $\mathrm{H}(r ; \theta)$ within the limit process are taken into account via the residue theorem. Calculating the arising residues using Leibniz' rule yields the $\theta$-exponential part (27). The limit of loop integrals is just the $(p, \theta)$-residual part (29a) and the proof of the decomposition (26) is complete.

It remains to prove the estimate (30). For sufficiently large $m$, the difference $\rho_{\mu ; p}^{\beta ; \delta}(\zeta)-\widetilde{\rho}_{\mu ; m}^{\beta}(\zeta)$ is a finite linear combination of complex powers $\zeta^{\gamma}$ with $\Re \gamma>p$. Therefore, using (19), the absolute value of the loop integral of $\mathrm{e}^{\xi \zeta} \zeta^{\delta}\left(\rho_{\mu ; p}^{\beta ; \delta}(\zeta)-\right.$ $\left.\widetilde{\rho}_{\mu ; m}^{\beta}(\zeta)\right)$ is found to be bounded by $C^{\prime}|\xi|^{-p-1}$ for all $\xi \in \mathbb{A} \mathbb{S}\left(r^{\prime},+\infty ; \theta^{\prime}\right)$ with $C^{\prime}<\infty$. Therefore, it suffices to estimate the loop integral of $\mathrm{e}^{\xi \zeta} \zeta^{\delta} \widetilde{\rho}_{\mu ; m}^{\beta}(\zeta)$ for some arbitrary but fixed $m \in \mathbb{N}$ instead. 
Choosing $m$ large enough guarantees that $\zeta^{\delta} \widetilde{\rho}_{\mu ; m}^{\beta}(\zeta)$ remains bounded for $|\zeta| \rightarrow 0$. Then the limit of loop integrals can be understood as an absolutely convergent integral over the limit contour $\mathrm{H}(0+; \theta)$. Substituting $\zeta \mapsto a_{\xi} \cdot \zeta$, where $a_{\xi}:=r^{\prime} /|\xi| \leq 1$, one obtains

$$
\begin{aligned}
& \left|\int_{\mathrm{H}(0+; \theta)} \mathrm{e}^{\xi \zeta} \zeta^{\delta} \widetilde{\rho}_{\mu ; m}^{\beta}(\zeta) \mathrm{d} \zeta\right| \leq \int_{\mathrm{H}(0+; \theta)} \mathrm{e}^{\Re \xi \zeta}\left|\zeta^{\delta} \widetilde{\rho}_{\mu ; m}^{\beta}(\zeta)\right| \mathrm{d}|\zeta| \\
& \quad=\left(\frac{r^{\prime}}{|\xi|}\right)^{p+1} \int_{\mathrm{H}(0+; \theta)} \mathrm{e}^{|\zeta| r^{\prime} \Re(\xi \zeta /|\xi \zeta|)} \cdot|\zeta|^{p} \cdot\left|\left(a_{\xi} \zeta\right)^{\delta-p} \widetilde{\rho}_{\mu ; m}^{\beta}\left(a_{\xi} \zeta\right)\right| \mathrm{d}|\zeta| .
\end{aligned}
$$

Finding a $\xi$-independent finite upper bound for the integral in the last equation will finish the proof. First, the absolute value $\left|\left(1-\Sigma \mu \zeta^{\beta}\right)^{-1}\right|$ remains bounded for $\zeta \in$ $\mathrm{H}(0+; \theta)$ because the function is continuous, finite-valued, due to $\theta \notin \arg \mathrm{Z}_{\mu}^{\beta}$, and tends to zero for $|\zeta| \rightarrow 0$ uniformly on any sector $\mathbb{S}\left(\theta^{\prime \prime}\right)$. Now, because $a_{\xi} \leq 1$ and $\Re(\xi \zeta /|\xi \zeta|) \leq \sin \left(\theta-\theta^{\prime}\right)<0$, it suffices to find a polynomial in $R \in \mathbb{R}_{+}$that is an upper bound for the function

$$
R \mapsto \sup \left\{\left|\zeta^{\delta-p}\left[\mu \zeta^{\beta}\right]^{m}\right|: \zeta=s \mathrm{e}^{ \pm \mathrm{i} \theta}, s \in(0, R]\right\}
$$

Using that $R^{b} \leq R^{a}+R^{c}$ for all $R \in \mathbb{R}_{+}, a, b, c \in \mathbb{R}$ with $a \leq b \leq c$ and the monotonicity of $\mathbb{R}_{+} \ni R \mapsto R^{a}$ for $a \in\{0\} \cup \mathbb{R}_{+}$one obtains

$$
\begin{aligned}
\left|\zeta^{\delta-p}\left[\mu \zeta^{\beta}\right]^{m}\right| & \leq \sum_{\Sigma q=m}[[q]] \cdot|\mu|^{q} \cdot\left|\zeta^{\delta-p+\beta q}\right| \\
& \leq C^{\prime \prime} \cdot R^{\Re \delta-p}\left(R^{m b_{+}}+R^{m b_{-}}\right)
\end{aligned}
$$

for all $\zeta=s \mathrm{e}^{ \pm \mathrm{i} \theta}, s \in(0, R]$ where $b_{+}:=\max \Re \beta, b_{-}:=\min \Re \beta$ and $C^{\prime \prime}<\infty$. Using (34) and Euler's integral formula for the Gamma function one estimates

$$
\begin{aligned}
& \int_{\mathrm{H}(0+; \theta)} \mathrm{e}^{|\zeta| r^{\prime} \Re(\xi \zeta /|\xi \zeta|)} \cdot|\zeta|^{p} \cdot\left|\left(a_{\xi} \zeta\right)^{\delta-p} \widetilde{\rho}_{\mu ; m}^{\beta}\left(a_{\xi} \zeta\right)\right| \mathrm{d}|\zeta| \\
& \quad \leq 2 C^{\prime} C^{\prime \prime} \mathrm{e}^{3 \pi|p| / 2} \int_{0}^{\infty} \mathrm{e}^{-r^{\prime} R \sin \left(\theta^{\prime}-\theta\right)} R^{p} R^{\Re \delta-p}\left(R^{m b_{-}}+R^{m b_{+}}\right) \mathrm{d} R \\
& \leq 2 C^{\prime} C^{\prime \prime} \mathrm{e}^{3 \pi|p| / 2}\left[\frac{\Gamma\left(\Re \delta+m b_{-}+1\right)}{\left(r^{\prime} \sin \left(\theta^{\prime}-\theta\right)\right)^{1+\Re \delta+m b_{-}}}+\frac{\Gamma\left(\Re \delta+m b_{+}+1\right)}{\left(r^{\prime} \sin \left(\theta^{\prime}-\theta\right)\right)^{1+\Re \delta+m b_{+}}}\right] .
\end{aligned}
$$

This expression is independent of $\xi$. Combining (32) and (35) yields (30).

Remark 1 In the case where $\beta$ consists of integers the function $1-\Sigma \mu \zeta^{\beta}$ can be interpreted as a meromorphic function on $\mathbb{C}$. If, in addition, $\delta \in \mathbb{N}$ then the loop in (22) can be deformed into a closed loop that encircles the zeros of $1-\Sigma \mu \zeta^{\beta}$ in $\mathbb{C}$. Therefore, one obtains $\mathrm{G}_{\mu ;+}^{\beta ; \delta}=\mathrm{E}_{\pi} \mathrm{G}_{\mu ;+}^{\beta ; \delta}$ and $\mathrm{G}_{\mu ;+}^{\beta ; \delta}$ is a finite sum of distributions of the form $\mathrm{p}_{+}^{k} \cdot \mathrm{e}^{a t}$ with $k \in \mathbb{N}_{1}, a \in \mathbb{C}$. This coincides with the well known formula 
for the causal fundamental solution of integer order differential equations on the real line.

Remark 2 In the case where $\left|\arg \mathrm{Z}_{\mu}^{\beta}\right|>\pi / 2$ one can choose $\theta$ such that $\left|\arg \mathrm{Z}_{\mu}^{\beta}\right|>\theta$. Here and in the following, for subsets $Z \subseteq \mathbb{L}$ and $\phi \in \mathbb{R}_{+}$, the notation $|\arg Z|>\phi$ always means that $|\zeta|>\phi$ for all $\zeta \in Z$. Then the $\theta$-exponential part vanishes and it holds $\mathrm{G}_{\mu ;+}^{\beta ; \delta}=\mathrm{P}^{p} \mathrm{G}_{\mu ;+}^{\beta ; \delta}+\mathrm{R}_{\theta}^{p} \mathrm{G}_{\mu ;+}^{\beta ;}$. The limit operation $r \searrow 0$ in (29a) is superflous in this case and the loop integral is independent of $r \in \mathbb{R}_{+}$. If, in addition, $\Re \delta>-1$ the loop $\mathrm{H}(r ; \theta)$ can be replaced by the limit loop $\mathrm{H}(0+; \theta)$. Note, that with $p$ chosen small enough, it holds $\mathrm{G}_{\mu ;+}^{\beta ; \delta}=\mathrm{R}_{\theta}^{p} \mathrm{G}_{\mu ;+}^{\beta ; \delta}$.

\section{Subalgebras of $\mathscr{F}+$ characterized by asymptotics}

Using Theorem 3 one obtains a characterization of the leading terms for $t \rightarrow+\infty$. Let $f, g: \mathbb{R} \rightarrow \mathbb{C}$. The expressions " $f(t) \preceq g(t)$ for $t \rightarrow+\infty$ " respectively " $f(t) \prec g(t)$ for $t \rightarrow+\infty$ " will be used to denote the asymptotic statements

$$
\begin{array}{ll}
\exists C \in \mathbb{R}_{+}, T \in \mathbb{R} \forall t \in[T,+\infty) & :|f(t)| \leq C|g(t)|, \\
\forall \in \in \mathbb{R}_{+} \exists T \in \mathbb{R} \forall t \in[T,+\infty) & :|f(t)| \leq \epsilon|g(t)| .
\end{array}
$$

The expression " $f(t) \sim g(t)$ for $t \rightarrow+\infty$ " is defined as " $f(t)-g(t) \prec g(t)$ for $t \rightarrow$ $+\infty$ " and means that $f(t)$ and $g(t)$ are asymptotically equivalent for $t \rightarrow+\infty$. A trigonometric log-polynomial is a function $g$ of the form

$$
g(t)=\Sigma c t^{\mathrm{i} b} \mathrm{e}^{\mathrm{i} \omega t} \quad \text { for } t \in(0,+\infty) \quad \text { with } b \in \mathbb{R}^{n}, c \in \mathbb{C}^{n}, \omega \in \mathbb{R}^{n}, n \in \mathbb{N} .
$$

For distinct tuples $\left(b_{k}, \omega_{k}\right)$ the coefficients $c$ are uniquely determined by $g(t)$. Evaluating now Theorem 3 for $t>0$ one obtains the following asymptotic characterizations:

Theorem 4 Let $n \in \mathbb{N}$ and $(\beta, \delta, \mu) \in \mathbb{H}^{n} \times \mathbb{C} \times \mathbb{C}^{n}$ with dominant order index. Let $a_{\mu}^{\beta ; \delta}$ denote the smallest $a \in \mathbb{R}$ with the property that for some $p \in \mathbb{R}$ it holds

$$
\mathrm{G}_{\mu ;+}^{\beta ; \delta}(t) \preceq t^{p-1} \mathrm{e}^{a t} \text { for } t \rightarrow+\infty .
$$

Let $p_{\mu}^{\beta ; \delta}$ denote the smallest $p \in \mathbb{R}$ such that (38) holds with $a=a_{\mu}^{\beta ; \delta}$. If $(\beta, \delta) \in \mathbb{N}^{n} \times \mathbb{N}$ or there exists $\zeta_{0} \in Z_{\mu}^{\beta}$ with $\left|\arg \zeta_{0}\right| \leq \pi / 2$, then

$$
\begin{aligned}
& a_{\mu}^{\beta ; \delta}=\sup \left\{\Re \zeta_{0}: \zeta_{0} \in Z_{\mu}^{\beta},\left|\arg \zeta_{0}\right| \leq \pi / 2\right\}, \\
& p_{\mu}^{\beta ; \delta}=\sup \left\{m_{\mu}^{\beta}\left(\zeta_{0}\right): \zeta_{0} \in Z_{\mu}^{\beta},\left|\arg \zeta_{0}\right| \leq \pi / 2, \Re \zeta_{0}=a_{\mu}^{\beta ; \delta}\right\} .
\end{aligned}
$$

Otherwise, it holds $a_{\mu}^{\beta ; \delta}=0$ and

$$
p_{\mu}^{\beta ; \delta}=\sup \left\{-\Re(\delta+\Sigma \beta q): q \in \mathbb{N}^{n}, \delta+\Sigma \beta q \notin \mathbb{N}\right\} .
$$


There exists a unique non-zero trigonometric log-polynomial $g_{\mu}^{\beta ; \delta}(t)$ such that

$$
\mathrm{G}_{\mu ;+}^{\beta ; \delta}(t) \sim t^{p-1} \mathrm{e}^{a t} g_{\mu}^{\beta ; \delta}(t) \quad \text { for } t \rightarrow+\infty \quad \text { where } a=a_{\mu}^{\beta ; \delta}, p=p_{\mu}^{\beta ; \delta} .
$$

If $\beta$ and $\delta$ are real, then $g_{\mu}^{\beta ; \delta}(t)$ is a trigonometric polynomial.

Lemma 1 Let $u$ be a distribution that is regular on $(0,+\infty)$.

1. For any $a, p \in \mathbb{R}$ and $\eta \in \mathscr{D}$ it holds that

$$
u(t) \preceq t^{p} \mathrm{e}^{a t} \text { for } t \rightarrow+\infty \quad \Rightarrow \quad(\eta * u)(t) \preceq t^{p} \mathrm{e}^{a t} \text { for } t \rightarrow+\infty .
$$

The same implication holds with $\preceq$ replaced by $\prec$.

2. Let $a, p \in \mathbb{R}$ and $g$ a non-zero trigonometric polynomial and assume

$$
u(t) \sim t^{p} \mathrm{e}^{a t} g(t) \quad \text { for } t \rightarrow+\infty .
$$

Then one finds $\phi_{1}, \ldots, \phi_{n} \in \mathscr{D}, n \in \mathbb{N}$ with the property that

$$
\left|\phi_{1} * u\right|(t)+\cdots+\left|\phi_{n} * u\right|(t) \succeq t^{p} \mathrm{e}^{a t} \quad \text { for } t \rightarrow+\infty .
$$

Proof Part 1: For real numbers $a, p \geq 0$ and $H \in \mathbb{R}_{+}$one estimates

$$
(1+t)^{p} \leq(1+t+h)^{p} \leq(1+H)^{p}(1+t)^{p}, \quad \mathrm{e}^{a t} \leq \mathrm{e}^{a(t+h)} \leq \mathrm{e}^{a H} \mathrm{e}^{a t}
$$

for all $t \in \mathbb{R}_{+}, 0 \leq h \leq H$. For $a, p \leq 0$ reverse these inequalities. Then (42) is proved using (44) and an estimate of the form $\left|\int_{0}^{\infty} \eta(s) f(s) \mathrm{d} s\right| \leq \int|\eta(s)| \mathrm{d} s$. $\sup _{s \in[t, t+H]}|f(s)|$.

Part 2: Let $\lambda \in \mathbb{R}_{+}$be $\leq$the smallest non-zero quarter wave length of the summands of $g$. Then let $\phi \in \mathscr{D}$ be positive, non-zero with support in $[0, \lambda]$. Due to (44) the inequality (43b) can be satisfied by choosing the $\phi_{1}, \ldots, \phi_{n}$ as suitable translates of $\phi$.

Theorem 5 Let $n \in \mathbb{N}$ and $(\beta, \delta, \mu) \in \mathbb{R}_{+}^{n} \times \mathbb{R} \times \mathbb{C}^{n}$.

1. It holds that $\mathrm{G}_{\mu ;+}^{\beta ; \delta} \in \mathscr{S}^{\prime}$ iff $\left|\arg \mathrm{Z}_{\mu}^{\beta}\right| \geq \pi / 2$.

2. It holds that $\mathrm{G}_{\mu ;+}^{\beta ; \delta} \in \mathscr{D}_{L^{1}}^{\prime}$ iff $\left|\arg \mathrm{Z}_{\mu}^{\beta}\right|>\pi / 2$ and $\delta \geq 0$.

3. It holds that $\mathrm{G}_{\mu ;+}^{\beta ; \delta} \in \mathscr{O}_{C}^{\prime}$ iff $\left|\arg \mathrm{Z}_{\mu}^{\beta}\right|>\pi / 2$ and $\beta$, $\delta$ are integer.

Proof Let $u:=\mathrm{G}_{\mu ;+}^{\beta ; \delta}$. Due to analogy it suffices to prove Part 1 .

If $\left|\arg Z_{\mu}^{\beta}\right| \geq \pi / 2$, then Theorem 4 and Lemma 1.1 imply that for $\phi \in \mathscr{D}$ one finds $n \in \mathbb{N}$ such that $|u * \phi|(t) \preceq t^{n}$ for $t \rightarrow+\infty$. Combining this with the fact that $|u * \phi|$ is a continuous function that vanishes for all $t \leq T$ for some fixed $T \in \mathbb{R}$ implies that $|u * \phi|$ is dominated by some polynomial on all of $\mathbb{R}$.

Assume that $u \in \mathscr{S}^{\prime}$, but that $\left|\arg \mathrm{Z}_{\mu}^{\beta}\right|<\pi / 2$. The assumption $u \in \mathscr{S}^{\prime}$ implies that $|\phi * u|$ is bounded by a polynomial and thus a finite sum of such terms is bounded 
by a polynomial. According to Theorem 4 and Lemma 1.2 one finds $\phi_{1}, \ldots, \phi_{n} \in \mathscr{D}$, $n \in \mathbb{N}$ such that (43b) holds with $a>0$. This is a contradiction.

The next Theorem provides the Laplace transforms of elements from $\mathscr{F}_{+}$. For basic definitions and results for the Laplace transform $\mathfrak{L}$, see [50, p.255,Eq.(2)]. The Fourier transform $\mathfrak{F} u(\omega)$ of a distribution $u \in \mathscr{D}_{L^{1}}^{\prime}$ is point-wise defined as $\mathfrak{F} u(\omega)=\left\langle u, \mathrm{e}^{\mathrm{i} \omega t}\right\rangle_{1}$ for all $\omega \in \mathbb{R}\left[36\right.$, Proposition 1.6.6(2)] where $\langle\cdot, \cdot\rangle_{1}$ denotes the pairing $\mathscr{D}_{L^{1}}^{\prime} \times \mathscr{B} \rightarrow \mathbb{C}$ (see Section 6). The pairing $\mathscr{S}^{\prime} \times \mathscr{S} \rightarrow \mathbb{C}$ is denoted by $\langle\cdot, \cdot\rangle_{\mathrm{s}}$ and $\langle\cdot, \cdot\rangle_{\mathscr{L}}$ is defined as in [50, p.255,eq.(3)]. The following Lemma connects Fourier and Laplace transforms.

Lemma 2 For $u \in \mathscr{D}_{L^{1}}^{\prime} \cap \mathscr{D}_{+}^{\prime}$ and $f \in \mathscr{B}$ it holds that $\lim _{\kappa}{ }_{0}\left\langle u, \mathrm{e}^{-\kappa t} f\right\rangle_{\mathscr{L}}=\langle u, f\rangle_{1}$.

Proof Let $\chi$ be a smooth function with $\chi(t)=1$ for $t \geq 0$ and support bounded on the left. Then it follows that

$$
\lim _{\kappa \searrow 0}\left\langle u, \mathrm{e}^{-\kappa t} f\right\rangle_{\mathscr{L}}=\lim _{\kappa \searrow 0}\left\langle u, \mathrm{e}^{-\kappa t} f \chi\right\rangle_{\mathrm{s}}=\lim _{\kappa \searrow 0}\left\langle u, \mathrm{e}^{-\kappa t} f \chi\right\rangle_{1}=\langle u, f \chi\rangle_{1}=\langle u, f\rangle_{1} .
$$

The first equality follows from the Definition. For the second equality see [4, p. 186]. The third equality follows because $\mathrm{e}^{-\kappa t} f \chi \rightarrow f \chi$ in $\mathscr{B}$ for $\kappa \searrow 0$. The fourth equality follows from $\chi \in \mathscr{B}$.

Definition 3 Let $u \in \mathscr{F}+$ and write $u$ in the form

$$
u=\frac{\Sigma \lambda \mathrm{q}_{+}^{\alpha}}{\delta-\Sigma \mu \mathrm{q}_{+}^{\beta}} \quad \text { with } \begin{gathered}
\alpha_{1}<\cdots<\alpha_{n}, \quad \lambda \in \mathbb{C}^{n}, \lambda_{k} \neq 0, n \in \mathbb{N} \\
0<\beta_{1}<\cdots<\beta_{m}, \mu \in \mathbb{C}^{m}, \mu_{k} \neq 0, m \in \mathbb{N}
\end{gathered}
$$

1. Define $\mathscr{G}_{+} \subseteq \mathscr{F}_{+}$by “ $\left|\arg Z_{\mu}^{\beta}\right| \geq \pi / 2$ implies $u \in \mathscr{G}_{+}$". ${ }^{2}$

2. Define $\mathscr{R}_{+} \subseteq \mathscr{F}_{+}$by " $\left|\arg Z_{\mu}^{\beta}\right|>\pi / 2$ and $\alpha_{1} \geq 0$ implies $u \in \mathscr{R}_{+}$".

3. Define $\mathscr{L}_{+} \subseteq \mathscr{F}_{+}$by “ $\left|\arg \mathrm{Z}_{\mu}^{\beta}\right|>\pi / 2$ and $\alpha, \beta$ integer implies $u \in \mathscr{L}_{+}$".

Theorem 6 Every $u \in \mathscr{F}_{+}$, written in the form (46), is Laplace transformable with abscissa of convergence $\sigma \leq a_{\mu}^{\beta ; 0}$ (see Theorem 4). The Laplace transform $\mathfrak{L} u$ extends meromorphically from $\mathbb{H}_{\sigma}$ to $\mathbb{L}$ and is given by

$$
\mathfrak{L} u(\zeta)=\frac{\Sigma \lambda \zeta^{\alpha}}{1-\Sigma \mu \zeta^{\beta}} \quad \text { for } \zeta \in \mathbb{L} .
$$

If $u \in \mathscr{G}_{+}$, then $\sigma \leq 0$. Further, if $u \in \mathscr{R}_{+}$, then $\mathfrak{L} u(s)$ extends continuously to the closed half-plane $\overline{\mathbb{H}}:=\{s \in \mathbb{C}: \Re s \geq 0\}$. Thus, the Fourier transform $\mathfrak{F} u: \mathbb{R} \rightarrow \mathbb{C}$ exists, is continuous and reads as

$$
\mathfrak{F} u(\omega)=\frac{\Sigma \lambda(\mathrm{i} \omega)^{\alpha}}{1-\Sigma \mu(\mathrm{i} \omega)^{\beta}} \quad \text { for } \omega \in \mathbb{R} \text { where } \mathrm{i} \omega=\widetilde{\mathrm{e}}^{ \pm \mathrm{i} \pi / 2} \omega \text { for } \omega \gtrless 0 \text {. }
$$

\footnotetext{
${ }^{2}$ More explicitly, $\mathscr{G}_{+}$consists of those $u \in \mathscr{F}+$ with the property that $u$ can be written in the form (46) with $\left|\arg Z_{\mu}^{\beta}\right| \geq \pi / 2$. Part 2 and Part 3 are interpreted in the same fashion.
} 
If $u \in \mathscr{L}_{+}$, then $\mathfrak{L} u$ extends to a meromorphic function on $\mathbb{C}$.

Proof The statement on Laplace transformability is clear. For $\delta+2<\max \beta$ the Hankel formula (22) can be transformed into the Laplace inversion formula

$$
\mathrm{G}_{\mu ;+}^{\beta ; \delta}(t)=\frac{1}{2 \pi \mathrm{i}} \lim _{r \rightarrow+\infty} \int_{-r}^{r} \frac{\zeta^{\delta}}{1-\Sigma \mu \zeta^{\beta}} \mathrm{e}^{t(\sigma+\epsilon+\mathrm{i} s)} \mathrm{d} s \quad \text { for } t, \epsilon \in \mathbb{R}_{+},
$$

by deforming the contour of integration $\mathrm{i} \mathbb{R}$ into a Hankel loop $\mathrm{H}(r ; \pi)$ with $r$ large enough. This is permitted because the integrand in (49) tends to zero faster than $|\zeta|^{\delta-\max \beta} \leq|\zeta|^{-2}$ for $|\zeta| \rightarrow+\infty$ when $\zeta \in \mathbb{A} S(r,+\infty ; \pi) \backslash \mathbb{A S}(r,+\infty ; \pi / 2)$, $r \geq 1$.

The distribution $\mathrm{G}_{\mu ;+}^{\beta ; \delta}$ has a continuous first derivative according to Proposition 2 and Eq. (17). Combining this with Eq. (49) and the fact that supp $\mathrm{G}_{\mu ;+}^{\beta ; \delta}=[0,+\infty)$ one concludes that the function $\zeta^{\delta} /\left(1-\Sigma \mu \zeta^{\beta}\right)$ is the Laplace transform of $\mathrm{G}_{\mu ;+}^{\beta ; \delta}$ [50, Ch. 8]. Now Eq. (47) follows using that $\mathfrak{L}\left(\mathrm{D}^{m} u\right)(s)=s^{m} \mathfrak{L}(u)(s)$ for $m \in \mathbb{N}$ and $s \in \mathbb{H}_{\sigma}$ and linearity.

The remaining statements are immediate from Lemma 2.

Theorem 7 The sets $\mathscr{G}_{+}, \mathscr{R}_{+}$and $\mathscr{L}_{+}$are subalgebras of $\mathscr{F}_{+}$that satisfy the inclusions $\mathscr{G}_{+} \subseteq \mathscr{S}^{\prime}, \mathscr{R}_{+} \subseteq \mathscr{D}_{L^{1}}^{\prime}$ and $\mathscr{L}_{+} \subseteq \mathscr{O}_{C}^{\prime}$ hold true.

Remark 3 We conjecture that Theorem 5 can be extended to general convolution quotients when appropriately reduced fractions are considered. Theorem 7 covers one implication of this extension.

\section{Distributional convolution modules}

This section describes a method to construct convolution modules of distributions for prescribed totally convolvable sets of distributions, see Definition 5 . These modules are maximal with respect to convolvability and characterized in Theorems 8 and 9 which are based on Propositions 4 and 5. The proofs for these propositions are of technical nature and merely $p$-ary variants to their binary or ternary variants, compare [35, Proposition 1.3.4]. A proof for Proposition 4 will be published elsewhere.

The results in this section are valid in arbitrary dimensions $d \in \mathbb{N}$ and the suffix $\left(\mathbb{R}^{d}\right)$ will be suppressed everywhere except within Definition 4 . Notions for convolvability and convolutes of distributions are taken from [34,35]. Let $\mathscr{B}$ denote the locally convex space of smooth functions with uniformly bounded derivatives. The topology on $\mathscr{B}$ is induced by the seminorms

$$
f \mapsto \sup \left\{\left\|g \cdot \mathrm{D}^{\alpha} f\right\|_{\infty}: \alpha \in \mathbb{N}^{d}, \Sigma \alpha \leq m\right\} \quad \text { for all } m \in \mathbb{N}, g \in \mathscr{C}_{0},
$$

where $\mathscr{C}_{0}$ is the space of continuous functions vanishing at infinity. The dual space of $\mathscr{B}$, the space of integrable distributions, is denoted by $\mathscr{D}_{L^{1}}^{\prime}$. The pairing $\mathscr{B} \times \mathscr{D}_{L^{1}}^{\prime} \rightarrow \mathbb{C}$ is denoted by $\langle\cdot, \cdot\rangle_{1}$. 
Definition 4 A tuple $\left(u_{1}, \ldots, u_{p}\right) \in\left(\mathscr{D}^{\prime}\left(\mathbb{R}^{d}\right)\right)^{p}, p \in \mathbb{N}$ is called convolvable if

$$
\phi^{\Delta p}\left(u_{1} \otimes \cdots \otimes u_{p}\right) \in \mathscr{D}_{L^{1}}^{\prime}\left(\mathbb{R}^{d p}\right) \quad \text { for all } \phi \in \mathscr{D}\left(\mathbb{R}^{d}\right),
$$

where $\phi^{\Delta p}\left(x_{1}, \ldots, x_{p}\right):=\phi\left(x_{1}+\cdots+x_{p}\right)$ denotes the $p$-codiagonal function associated to $\phi$. Then, the convolute $u_{1} * \cdots * u_{p}$ is defined as

$$
\left\langle\phi, u_{1} * \ldots * u_{p}\right\rangle:=\left\langle 1, \phi^{\Delta p}\left(u_{1} \otimes \cdots \otimes u_{p}\right)\right\rangle_{1} \quad \text { for all } \phi \in \mathscr{D}\left(\mathbb{R}^{d}\right) .
$$

A set of distributions $U \subseteq \mathscr{D}^{\prime}\left(\mathbb{R}^{d}\right)$ will be called totally convolvable whenever all tuples $\left(u_{1}, \ldots, u_{p}\right)$ with $u_{1}, \ldots, u_{p} \in U$ and $p \in \mathbb{N}$ are convolvable.

Due to the symmetric nature of the definition it is clear that the notions of convolvability and convolute of a given tuple of distributions are invariant under arbitrary permutations of that tuple. Further, it is clear that the set of convolvable $p$-tuples is linear and that the mapping $\left(u_{1}, \ldots, u_{p}\right) \mapsto u_{1} * \cdots * u_{p}$ is multilinear.

Convolution of $\overline{\mathbb{R}}_{+}$-valued lower semicontinuous functions is seen to be associative without further restrictions by virtue of the Lebesgue-Fubini theorem [8, p. 54f]. The question under which circumstances the associative law holds for convolution of distributions is less obvious (and suprisingly rarely treated in the literature). The answer in Proposition 5 below will provide a satisfactory sufficient condition.

Proposition 4 Let $p \in \mathbb{N}, u_{1}, \ldots, u_{p} \in \mathscr{D}^{\prime}$. Then $\left(u_{1}, \ldots, u_{p}\right)$ is convolvable iff

$$
\forall \phi_{1}, \ldots, \phi_{p} \in \mathscr{D}, x \in \mathbb{R}^{d} \quad: \quad\left(\left|\phi_{1} * u_{1}\right| * \cdots *\left|\phi_{p} * u_{p}\right|\right)(x)<\infty .
$$

Remark 4 The binary variant of this Proposition is just Chevalley's convolvability condition [35] in disguise which reads as $(\phi * u) \cdot(\psi * v)^{\vee} \in L^{1}$ for all $\phi, \psi \in \mathscr{D}$.

Proposition 5 Let $p \in \mathbb{N}$ and $u_{1}, \ldots, u_{p} \in \mathscr{D}^{\prime}$, each $u_{k}$ non-zero. Then, if $\left(u_{1}, \ldots, u_{p}\right)$ is convolvable, brackets can be introduced arbitrarily into the expression $u_{1} * \cdots * u_{p}$ without creating non-convolvable tuples or changing the result of this expression.

Definition 5 The maximal distributional convolution module associated to a totally convolvable set $A \subseteq \mathscr{D}^{\prime}$ is defined as

$$
(A)_{\mathscr{D}^{\prime}}^{* \mathrm{M}}:=\left\{m \in \mathscr{D}^{\prime} \mid \forall p \in \mathbb{N}, a_{1}, \ldots, a_{p} \in A:\left(a_{1}, \ldots, a_{p}, m\right) \text { is convolvable }\right\} .
$$

Theorem 8 Let $A \subseteq \mathscr{D}^{\prime}$ be totally convolvable and such that $A * A \subseteq A$. Convolution defines bilinear operations

$$
*: \mathbb{C}[A] \times \mathbb{C}[A] \rightarrow \mathbb{C}[A], \quad *: \mathbb{C}[A] \times(A)_{\mathscr{D}^{\prime}}^{* \mathrm{M}} \rightarrow(A)_{\mathscr{D}^{\prime}}^{* \mathrm{M}}
$$

that are associative. That is, for all $a, b, c \in A$ and $m \in(A)_{\mathscr{D}^{\prime}}^{* \mathrm{M}}$ it holds that

$$
a *(b * c)=(a * b) * c, \quad a *(b * m)=(a * b) * m
$$


Proof Total convolvability and the definition of $(A)_{\mathscr{D}^{\prime}}^{* \mathrm{M}}$ imply that the mappings $*: \mathbb{C}[A] \times \mathbb{C}[A] \rightarrow \mathbb{C}[A]$ and $*: \mathbb{C}[A] \times(A)_{\mathscr{D}^{\prime}}^{* \mathrm{M}} \rightarrow \mathscr{D}^{\prime}$ are well-defined and bilinear. Proposition 5 guarantees that the associative laws hold once it is proved that $\mathbb{C}[A] *(A)_{\mathscr{D}^{\prime}}^{* \mathrm{M}} \subseteq(A)_{\mathscr{D}^{\prime}}^{* \mathrm{M}}$.

Let $a_{1}, \ldots, a_{p}, b \in A$ and $m \in(A)_{\mathscr{D}}^{* \mathrm{M}}$ be non-zero, $p \in \mathbb{N}$. The tuple of distributions $\left(a_{1}, \ldots, a_{p}, b, m\right)$ is convolvable by definition. Proposition 5 implies that $\left(a_{1}, \ldots, a_{p}, b * m\right)$ is convolvable as well which means that $b * m \in(A)_{\mathscr{D}^{\prime}}^{* \mathrm{M}}$.

Definition 6 The $L^{1}$-weighted space of distributions associated to a set of lower semicontinuous functions $I \subseteq \mathscr{I}$ is

$$
\mathscr{D}_{L^{1}(I)}^{\prime}:=\left\{u \in \mathscr{D}^{\prime} \mid \forall f \in I, \phi \in \mathscr{D}:\|f(\phi * u)\|_{1}<\infty\right\}
$$

where $\|g\|_{1}:=\int_{\mathbb{R}^{d}}|g(x)| \mathrm{d} x$.

Proposition 6 Let $I, J \subseteq \mathscr{I}$.

1. If all elements of I are locally bounded then $\mathscr{E}^{\prime} \subseteq \mathscr{D}_{L^{1}(I)}^{\prime}$.

2. The space $\mathscr{D}_{L^{1}(I)}^{\prime}$ is translation invariant.

3. If for all $f \in I$ there exist $g_{1}, \ldots, g_{n} \in J, x_{1}, \ldots, x_{n} \in \mathbb{R}^{d}, n \in \mathbb{N}, C \in \mathbb{R}_{+}$such that $f \leq C \sup \left\{\mathrm{T}_{x_{1}} g_{1}, \ldots, \mathrm{T}_{x_{n}} g_{n}\right\}$ then the inclusion $\mathscr{D}_{L^{1}(J)}^{\prime} \subseteq \mathscr{D}_{L^{1}(I)}^{\prime}$ holds.

The following summarizes some evident properties of the spaces $\mathscr{D}_{L^{1}(I)}^{\prime}$.

Theorem 9 Let A be totally convolvable and define the set

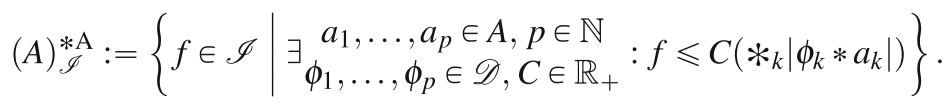

The maximal convolution module of $A$ is given by the weighted space

$$
(A)_{\mathscr{D}^{\prime}}^{* \mathrm{M}}=\mathscr{D}_{L^{1}(I)}^{\prime} \text { with } I=(\check{A})_{\mathscr{I}}^{* \mathrm{~A}} \text {. }
$$

Proof Let $f=\left|\check{\phi}_{1} * \check{a}_{1}\right| * \cdots *\left|\check{\phi}_{p} * \check{a}_{p}\right|$ with $\phi_{1}, \ldots, \phi_{p} \in \mathscr{D}, a_{1}, \ldots, a_{p} \in A$, $p \in \mathbb{N}$. Using that the associative law holds for arbitrary convolutes of $\overline{\mathbb{R}}_{+}$-valued lower semicontinuous functions one calculates for $x \in \mathbb{R}^{d}$ that

$$
\begin{aligned}
\left\|f\left(\mathrm{~T}_{-x} \phi * u\right)\right\|_{1} & =(|\check{f}| *|\phi * u|)(x) \\
& =\left(\left|\phi_{1} * a_{1}\right| * \cdots *\left|\phi_{p} * a_{p}\right| *|\phi * u|\right)(x) .
\end{aligned}
$$

Finiteness of the latter expression for all $\phi_{1}, \ldots, \phi_{p} \in \mathscr{D}, \phi \in \mathscr{D}, a_{1}, \ldots, a_{p} \in A$ and $x \in \mathbb{R}^{d}$ is equivalent to $u \in(A)_{\mathscr{D}^{\prime}}^{* \mathrm{M}}$ by Proposition 4 . 


\section{Distribution modules for fractional calculus}

Definitions and results for maximal distributional convolution modules from the previous section are now applied to $\mathscr{F}_{+}$and its subalgebras $\mathscr{G}_{+}, \mathscr{R}_{+}$and $\mathscr{L}_{+}$.

Definition 7 Let $p \in \mathbb{R}$. Define the sets

$$
\begin{aligned}
& P_{+}^{p}:=\left\{f \in \mathscr{I}_{+} \mid \exists C \in \mathbb{R}_{+} \forall t \in \mathbb{R}: f(t) \leq C(1+|t|)^{p}\right\} \\
& E_{+}^{a}:=\left\{f \in \mathscr{I}_{+} \mid \exists C \in \mathbb{R}_{+} \forall t \in \mathbb{R}: f(t) \leq C \mathrm{e}^{a t}\right\}
\end{aligned}
$$

and their reflected variants $P_{-}^{p}:=\left(P_{+}^{p}\right)^{\vee}$. Further, let

$$
E_{ \pm}:=\bigcup_{a \in \mathbb{R}} E_{ \pm}^{a}, \quad P_{ \pm}:=\bigcup_{p \in \mathbb{R}} P_{ \pm}^{p}, \quad Q_{ \pm}:=\bigcup_{p \in \mathbb{R}_{+}} P_{ \pm}^{-p-1}, \quad E_{ \pm}^{<0}:=\bigcup_{a \in \mathbb{R}_{+}} E_{ \pm}^{-a}
$$

Proposition 7 Let $a \in \mathbb{R}$ and $q \in \mathbb{R}_{+}$. Each of the sets $E_{ \pm}, P_{ \pm}, Q_{ \pm}$and $E_{ \pm}^{<0}$ is closed with respect to convolution.

Proof It suffices to prove that $P_{+}^{p-1} * P_{+}^{q-1} \subseteq P_{+}^{p+q-1}$ and $P_{+}^{-p-1} * P_{+}^{-p-1} \subseteq P_{+}^{-p-1}$ for $p, q \in \mathbb{R}_{+}$due to $\left(\mathrm{e}^{a t} f\right) *\left(\mathrm{e}^{a t} g\right)=\mathrm{e}^{a t}(f * g)$ and $\check{f} * \check{g}=(f * g)^{\vee}, a \in \mathbb{R}$, $f, g \in \mathscr{I}$ and because the property $S * S \subseteq S$ is preserved under directed unions. Using that convolution is isotone, homogeneous and translation invariant and observing that every element of $P_{+}^{p-1}$ is bounded by a translate of $C \mathrm{p}_{+}^{p}(t)$ for some $C \in \mathbb{R}_{+}$one concludes $P_{+}^{p-1} * P_{+}^{q-1} \subseteq P_{+}^{p+q-1}$ from the index law (10). For $p \in \mathbb{R}_{+}$one estimates

$$
\begin{aligned}
& \int_{0}^{t}(1+t-s)^{-p-1}(1+s)^{-p-1} \mathrm{~d} s=2 \int_{0}^{t / 2}((1+t-s)(1+s))^{-p-1} \mathrm{~d} s \\
& \leq 2(1+t / 2)^{-p-1} \int_{0}^{+\infty}(1+s)^{-p-1} \mathrm{~d} s \leq \frac{2^{p+2}}{p}(1+t)^{-p-1} .
\end{aligned}
$$

Combining this with arguments as above one obtains $P_{+}^{-p-1} * P_{+}^{-p-1} \subseteq P_{+}^{-p-1}$.

Theorem 10 The following results for maximal convolution modules hold:

$$
\begin{aligned}
\left(\mathscr{F}_{+}\right)_{\mathscr{D}^{\prime}}^{* \mathrm{M}}=\mathscr{D}_{L^{1}\left(E_{-}\right)}^{\prime} \\
\left(\mathscr{P}_{+}\right)_{\mathscr{D}^{\prime}}^{* \mathrm{M}}=\left(\mathscr{P}_{+}^{\mathbb{C}}\right)_{\mathscr{D}^{\prime}}^{* \mathrm{M}}=\left(\mathscr{G}_{+}\right)_{\mathscr{D}^{\prime}}^{* \mathrm{M}}=\mathscr{D}_{L^{1}\left(P_{-}\right)}^{\prime} \\
\left(\mathscr{Q}_{+}\right)_{\mathscr{D}^{\prime}}^{* \mathrm{M}}=\left(\mathscr{Q}_{+}^{\mathbb{H}}\right)_{\mathscr{D}^{\prime}}^{* \mathrm{M}}=\left(\mathscr{R}_{+}\right)_{\mathscr{D}^{\prime}}^{* \mathrm{M}}=\mathscr{D}_{L^{1}\left(Q_{-}\right)}^{\prime} \\
\left(\mathscr{L}_{+}\right)_{\mathscr{D}^{\prime}}^{* \mathrm{M}}=\mathscr{D}_{L^{1}\left(E_{-}^{<0}\right)}^{\prime}
\end{aligned}
$$

Proof Due to analogy if suffices to prove (61a). Using Lemma 1 and Theorem 4 one obtains that $\left|\mathscr{D} * \mathscr{F}_{+}\right| \subseteq E_{+}$and that every element of $E_{+}$is dominated by a sum of elements from $\left|\mathscr{D} * \mathscr{F}_{+}\right|$. Combining this with Proposition 7 one concludes 
that $\left(\left(\mathscr{F}_{+}\right)^{\vee}\right)_{\mathscr{I}}^{* \mathrm{~A}}=E_{-}$(see Eq. $\left.(56 \mathrm{~b})\right)$. With this equation the first statement is a consequence of Theorems 1 and 9.

For any subalgebra $\mathscr{A}$ of $\mathscr{D}_{+}^{\prime}$ let $(\mathscr{A})^{\times}$denote the set of distributions $a \in \mathscr{A}$ that are invertible as elements of $\left(\mathscr{D}_{+}^{\prime},+, *\right)$ and obey $a^{*-1} \in \mathscr{A}$.

Corollary 1 The convolution groups $\left(\mathscr{F}_{+}\right)^{\times},\left(\mathscr{G}_{+}\right)^{\times},\left(\mathscr{R}_{+}\right)^{\times}$resp. $\left(\mathscr{L}_{+}\right)^{\times}$operate bijectively and lineary on the spaces $\mathscr{D}_{L^{1}\left(E_{-}\right)}^{\prime}, \mathscr{D}_{L^{1}\left(P_{-}\right)}^{\prime}, \mathscr{D}_{L^{1}\left(Q_{-}\right)}^{\prime}$ resp. $\mathscr{D}_{L^{1}\left(E_{-}^{<0}\right)}^{\prime}$

Proposition 8 Let $u \in \mathscr{F}+$ be non-zero and write $u$ in the form

$$
u=\mathrm{q}_{+}^{\gamma} * \frac{\delta-\Sigma \lambda \mathrm{q}_{+}^{\alpha}}{\delta-\Sigma \mu \mathrm{q}_{+}^{\beta}} \quad \text { with } \gamma \in \mathbb{R}, \begin{aligned}
& 0<\alpha_{1}<\cdots<\alpha_{n}, \lambda \in \mathbb{C}_{\neq 0}^{n}, n \in \mathbb{N}, \\
& 0<\beta_{1}<\cdots<\beta_{m}, \mu \in \mathbb{C}_{\neq 0}^{m}, m \in \mathbb{N} .
\end{aligned}
$$

1. If $\left|\arg Z_{\lambda}^{\alpha}\right| \geq \pi / 2$ and $\left|\arg Z_{\mu}^{\beta}\right| \geq \pi / 2$ then $u \in\left(\mathscr{G}_{+}\right)^{\times}$.

2. If $\left|\arg Z_{\lambda}^{\alpha}\right|,\left|\arg Z_{\mu}^{\beta}\right|>\pi / 2$ and $\gamma=0$ then $u \in\left(\mathscr{R}_{+}\right)^{\times}$.

3. If $\left|\arg Z_{\lambda}^{\alpha}\right|,\left|\arg Z_{\mu}^{\beta}\right|>\pi / 2, \gamma=0$ and $\alpha, \beta$ are integer then $u \in\left(\mathscr{L}_{+}\right)^{\times}$.

Proof This is immediate from Definition 3.

\section{Conclusions for fractional calculus}

The final Section discusses consequences for fractional calculus and fractional differential equations that result from the previous sections.

\subsection{Extending domains of fractional derivatives and integrals}

From Eq. (61b) in Theorem 10 one obtains a generalization for the operators $\mathrm{D}_{-}^{\alpha}$, $\alpha \in \mathbb{C}$ from functions [31,32] to distributions. Let $\mathscr{E}=\mathscr{E}\left(\mathbb{R}_{+}\right)$be the space of smooth functions on $\mathbb{R}_{+}$with derivatives vanishing rapidly for $t \rightarrow+\infty$. The restriction to the half-axis is unnecessary, and all definitions and results are readily transported to the operators $\mathrm{D}_{+}^{\alpha}$ and the space $\widetilde{\mathscr{E}}=\widetilde{\mathscr{E}}(\mathbb{R})$ of smooth functions on $\mathbb{R}$ vanishing rapidly for $t \rightarrow-\infty$. With this extension one obtains bijective linear operators that commute with translations and obey the index law in full generality, that is

$$
\mathrm{D}_{+}^{\alpha}\left(\mathrm{D}_{+}^{\beta} f\right)=\mathrm{D}_{+}^{\alpha+\beta} f \quad \text { for all } \alpha, \beta \in \mathbb{C}, f \in \widetilde{\mathscr{E}} .
$$

It has been shown recently by the authors [19], that the space $\widetilde{\mathscr{E}}$ can be endowed with a weighted topology in a natural way such that one obtains bicontinous operators.

By means of Theorem 10, Eq. (61b), domain and range can now be enlarged to the space $\mathscr{D}_{L^{1}\left(P_{-}\right)}^{\prime}$ of distributions vanishing rapidly for $t \rightarrow-\infty$. Bijectivity, linearity and commutativity with translations are preserved under this extension. It contains earlier extensions that used exponentially weighted spaces of distributions [41], thus, more rapidly falling distributions. A rigorous treatment of the question of continuity is more complicated and requires to introduce new topologies. 
An even more fundamental extension to fractional calculus is obtained from Eq. (61c) of Theorem 10. The statement there shows that the space

$$
\mathscr{D}_{L^{1}\left(Q_{-}\right)}^{\prime}=\left\{\left.u \in \mathscr{D}^{\prime}\left|\forall p \in \mathbb{R}_{+}, \phi \in \mathscr{D}: \int_{-\infty}^{-1}\right|(\phi * u)(t)|\cdot| t\right|^{p-1} \mathrm{~d} t<+\infty\right\}
$$

can be assigned as joint domain and range to the operators $\mathrm{D}_{+}^{\alpha}, \alpha \in \mathbb{H}$ when they are defined as $u \mapsto \mathrm{q}_{+}^{\alpha} * u$ by means of Definition 4 . With this extension bijectivity is lost, but still, one has linear operators at hand that satisfy the index law and commute with translations. Comparing with the domain for Marchaud derivatives, as in [39, p.109], it is seen that the present extension has a similar growth condition, but for distributions instead of functions.

One value of the extension lies in the fact that $\mathscr{D}_{L^{1}\left(Q_{-}\right)}^{\prime}$ contains the space of uniformly bounded distributions $\mathscr{B}^{\prime}$, and thus, the space of periodic distributions. For example, the fractional derivatives of the functions $\varpi_{\mathrm{i} \omega}(t)=\exp (\mathrm{i} \omega t), t \in \mathbb{R}$ and the reversed Heaviside function $\Theta_{-}=1-\Theta_{+}$are defined. The section concludes with the calculation of these fundamental fractional derivatives.

Proposition 9 For $\alpha \in \mathbb{H}$ and $\sigma \in \overline{\mathbb{H}}$ holds $\mathrm{q}_{+}^{\alpha} * \varpi_{\sigma}=\sigma^{\alpha} \varpi_{\sigma}$ where $\varpi_{\sigma}(t)=\mathrm{e}^{\sigma t}$.

Proof Let $m \in \mathbb{N}$ such that $m-\Re \alpha>0$ and set $\beta:=m-\alpha$. First, assume $\Re \sigma>0$. Then $\varpi_{\sigma} \in \mathscr{D}_{L^{1}\left(P_{-}\right)}^{\prime}$ and one calculates that $\mathrm{q}_{+}^{\beta} * \varpi_{\sigma}=\sigma^{-\beta} \varpi_{\sigma}$. Using the associative law, which is justified by Eq. (61b) and Theorem 8, one obtains

$$
\mathrm{q}_{+}^{\alpha} * \varpi_{\sigma}=\mathrm{D}^{m} \mathrm{p}_{+}^{\beta} * \varpi_{\sigma}=\mathrm{p}_{+}^{\beta} * \mathrm{D}^{m} \varpi_{\sigma}=\sigma^{m}\left(\mathrm{p}_{+}^{\beta} * \varpi_{\sigma}\right)=\sigma^{m-\beta} \varpi_{\sigma}=\sigma^{\alpha} \varpi_{\sigma} .
$$

An application of Lemma 2 extends this result to $\sigma \in \overline{\mathbb{H}}$.

Corollary 2 It holds that $\mathrm{D}_{+}^{\alpha} \Theta_{-}=-\mathrm{p}_{+}^{1-\alpha}$ for all $\alpha \in \mathbb{H}$.

Proof Using Proposition 9 with $\sigma=0$ and that $\Theta_{-} \in \mathscr{D}_{L^{1}\left(Q_{-}\right)}^{\prime}$ one obtains

$$
\mathrm{D}_{+}^{\alpha} \Theta_{-}=\mathrm{q}_{+}^{\alpha} * \Theta_{-}=\mathrm{q}_{+}^{\alpha} *\left(1-\mathrm{p}_{+}^{1}\right)=\mathrm{q}_{+}^{\alpha} * 1-\mathrm{q}_{+}^{\alpha} * \mathrm{p}_{+}^{1}=-\mathrm{p}_{+}^{1-\alpha}
$$

proving the corollary.

The operators $\mathrm{p}_{+}^{\alpha}$ and $\mathrm{q}_{+}^{\alpha}$ from the field $\mathscr{F}_{+}$acting on the convolution module $\mathscr{D}_{L^{1}\left(E_{-}\right)}^{\prime}$ resemble the operators $J^{\alpha}$ and $S^{\alpha}$ obtained via the operational method in [13]. However, the operators $S^{\alpha}$ from [13] are constructed as members of a quotient field $\mathscr{M}_{-1}$ of a convolution ring $\left(C_{-1}, \circ,+\right)$ of functions on the right half axis. In the present work the quotient field $\mathscr{F}_{+}$is constructed by adjoining the inverses from the convolution algebra $\left(\mathscr{D}_{+}^{\prime}, *,+\right)$. It is at present not clear whether all hyperfunctions in $\mathscr{M}_{-1}$ can be interpreted as distributions, or how to extended the operators $S^{\alpha}$ to functions on the real line. 


\subsection{General linear fractional differential equations}

The most general linear response equation that can be formulated within the present framework of fractional derivatives reads as

$$
\eta \mathrm{D}_{+}^{\gamma}\left[u-\Sigma \lambda \mathrm{D}_{+}^{\alpha} u\right]=v-\Sigma \mu \mathrm{D}_{+}^{\beta} v \quad \text { for } u, v \in \mathscr{D}_{L^{1}\left(E_{-}\right)}^{\prime},
$$

with parameters $\alpha \in \mathbb{R}_{+}^{n}, \beta \in \mathbb{R}_{+}^{m}, \gamma \in \mathbb{R}, \lambda \in \mathbb{C}_{\neq 0}^{n}, \mu \in \mathbb{C}_{\neq 0}^{m}, \eta \in \mathbb{C}_{\neq 0}$ and $n, m \in \mathbb{N}$. In the case $m=0$ the right hand side reduces to $v$. According to Theorem 10 the operators $\eta \mathrm{D}_{+}^{\gamma}\left[1-\Sigma \lambda \mathrm{D}_{+}^{\alpha}\right]$ and $1-\Sigma \mu \mathrm{D}_{+}^{\beta}$ define automorphisms of $\mathscr{D}_{L^{1}\left(E_{-}\right)}^{\prime}$. This implies existence and uniqueness of solutions $u$ ("responses") for prescribed $v$ ("excitations"). Note, that the roles of $u$ and $v$ can be interchanged.

Under certain assumptions on the parameters it is possible to enlarge the domains. For example, under the assumptions

$$
\gamma=0, \begin{aligned}
& 0<\alpha_{1}<\cdots<\alpha_{n} \leq 1, \lambda=-\tau, \text { with } \tau \in \mathbb{R}_{+}^{n} \\
& 0<\beta_{1}<\cdots<\beta_{m} \leq 1, \mu=-\sigma, \text { with } \sigma \in \mathbb{R}_{+}^{m}
\end{aligned}
$$

it is guaranteed that $\left|\arg Z_{\lambda}^{\alpha}\right|,\left|\arg Z_{\mu}^{\beta}\right|>\pi / 2$. This follows from the inequality

$$
\Re \zeta^{\delta} \geq 0 \text { for all } \zeta \in \mathbb{L} \text { with }|\arg \zeta| \leq \pi / 2 \text { and } \delta \in(0,1] \text {. }
$$

According to Definition 3 and Eq. (61c) of Theorem 10 one obtains that the domain for $u$ and $v$ can be enlarged to $\mathscr{D}_{L^{1}\left(Q_{-}\right)}^{\prime}$ while preserving existence and uniqueness of solutions. For the case $m=0$, Theorem 5 proves that the condition $\left|\arg Z_{\lambda}^{\alpha}\right|>\pi / 2$ is also necessary to extend the domains to $\mathscr{D}_{L^{1}\left(Q_{-}\right)}^{\prime}$.

The case $\gamma=0$ and $m=0$ was discussed in [1] with the space $\mathscr{S}_{+}^{\prime}$ as domain for $u$ and $v$. In Theorem 3.2 from [1] existence and uniqueness were characterized in terms of $Z_{\lambda}^{\alpha}$. Note, that in the proof of [1, Theorem 3.2] it was stated that if the function $1-\Sigma \mu \zeta^{\alpha}$ has no zeros $\zeta_{0}$ with $\left|\arg \zeta_{0}\right|<\pi / 2$, then all of its zeros with $\left|\arg \zeta_{0}\right|<\pi$ fulfill $\left|\arg \zeta_{0}\right|=\pi / 2$. This is false, a counter example is given by $1+\zeta^{3 / 2}$. Nevertheless Theorem 3.2 from [1] is equivalent to Part 1 of Theorem 5 from the present work. In the notation of the present work it states that the problem $\eta\left[u-\Sigma \mu \mathrm{D}_{+}^{\alpha} u\right]=v$ with $u, v \in \mathscr{S}_{+}^{\prime}$ is well-posed with existence and uniqueness of solutions $u$ to prescribed $v$ if and only if $\left|\arg Z_{\lambda}^{\alpha}\right| \leq \pi / 2$. Theorem 10 from the present work extends this from $\mathscr{S}_{+}^{\prime}$ to the larger space $\mathscr{D}_{L^{1}\left(P_{-}\right)}^{\prime}$. 


\section{Application to dielectric relaxation}

\subsection{Composite fractional relaxation model extended to the full axis}

The composite fractional relaxation model for the dielectric relaxation function $f(t)$, $t>0[10]$

$$
\tau_{1} \mathrm{D}^{1} f+\tau_{2}^{\alpha} \mathrm{D}_{0+}^{\alpha, \mu} f+f=h \quad \text { with } \lim _{t \rightarrow 0} f(t)=f(0+)=f_{0+},
$$

involves the fractional derivative $\mathrm{D}_{0+}^{\alpha, \mu}$ with order $\alpha$ and type $\mu$ [10, Eq. (7)]. The parameters are $0<\tau_{1}, \tau_{2}<\infty, 0<\alpha<1,0 \leq \mu \leq 1$. In [10] the right hand side $h=0$ vanishes, the function $f$ is a $\mathscr{C}^{1}$-function defined on the half axis $(0, \infty)$, and interpreted as the normalized relaxation function after a constant external force is suddenly released at $t=0$. The purpose of this section is to extend Eq. (70) to the full axis and use it as a template for an application of Eq. (67) to dielectric relaxation in glasses.

Following [10] and [11] the Laplace transform $\mathfrak{L}_{+} \chi_{+}$of the dielectric response $\chi_{+}(t)$ on $t>0$ for the case $h=0$ is calculated as (cf. [11, Eq.(3)])

$$
\frac{1}{f_{0+}}\left(\mathfrak{L}_{+} \chi_{+}\right)(\zeta)=\frac{1+\left(\tau_{2} \zeta\right)^{\alpha}+\mathrm{r}_{\mu}^{\alpha}\left(\tau_{2} \zeta\right)^{1+\mu(\alpha-1)}}{1+\left(\tau_{2} \zeta\right)^{\alpha}+\tau_{1} \zeta}, \quad \zeta \in \mathbb{L}
$$

where $r_{\mu}^{\alpha}$ is a parameter. In the following $r_{\mu}^{\alpha} \geq 0$ will be assumed except for $r_{1}^{\alpha}=-1$. Equation (71) admits continuous extensions from $\zeta \in \mathbb{H} \subseteq \mathbb{L}$ to $\zeta \in \overline{\mathbb{H}}$.

The response function $\chi(t)$ is extended as $\chi(t)=\chi_{+}(t)+\chi^{\infty} \delta(t)$ to the full axis, where $\chi_{+}(t)$ is extended as $\chi_{+}(t)=0$ to $t \leq 0$. The relaxation function $f$ is extended discontinuously as $f(t)=f(0)=f_{0}=f_{0+}+\chi^{\infty}$ to $t \leq 0$. It can be written as

$$
\begin{aligned}
f(t)=\int_{t}^{\infty} \chi(s) \mathrm{d} s & =\chi^{\infty} \int_{t}^{\infty} \delta(s) \mathrm{d} s+f_{0+}-\int_{0}^{t} \chi_{+}(s) \mathrm{d} s \\
& =\chi^{\infty} \Theta_{-}(t)+f_{0+}-\left[\chi_{+} * \mathrm{p}_{+}^{1}\right](t)
\end{aligned}
$$

with $f_{0+}=\int_{0}^{\infty} \chi_{+}(t) \mathrm{d} t$. Using Eq. (71) as guidance the normalized response $\chi_{+} / f_{0+}$ is represented as a convolution quotient

$$
\widehat{\chi}=\frac{\chi-\chi^{\infty} \delta}{f_{0+}}=\frac{\chi_{+}}{f_{0+}}=\frac{\delta+\tau_{2}^{\alpha} \mathrm{q}_{+}^{\alpha}+\mathrm{r}_{\mu}^{\alpha} \tau_{2}^{1+\mu(\alpha-1)} \mathrm{q}_{+}^{1+\mu(\alpha-1)}}{\delta+\tau_{2}^{\alpha} \mathrm{q}_{+}^{\alpha}+\tau_{1} \mathrm{q}_{+}^{1}}
$$

by virtue of Theorem 6 and Definition 3 . The convolution quotient falls under case 2 of Proposition 8 and hence $\widehat{\chi} \in\left(\mathscr{R}_{+}\right)^{\times}$. Equation (61c) of Theorem 10 guarantees that the fractional differential equation

$$
u+\tau_{2}^{\alpha} \mathrm{D}_{+}^{\alpha} u+\tau_{1} \mathrm{D} u=w+\tau_{2}^{\alpha} \mathrm{D}_{+}^{\alpha} w+r_{\mu}^{\alpha} \tau_{2}^{1+\mu(\alpha-1)} \mathrm{D}_{+}^{1+\mu(\alpha-1)} w,
$$


is well defined with existence and uniqueness for all $u, w \in \mathscr{D}_{L^{1}\left(Q_{-}\right)}^{\prime}$, because it is a special case of (67) with (68). Equation (74) is equivalent to $u=\widehat{\chi} * w$ with $\widehat{\chi} \in\left(\mathscr{R}_{+}\right)^{\times}$a bijective convolution operator on $\mathscr{D}_{L^{1}\left(Q_{-}\right)}^{\prime}$. Equation (74) generalizes the fractional relaxation Eq. (70) from the positive half axis to the full real axis in a translation invariant manner. It has the form of Eq. (67) and is taken in Sect. 9.3 as a mathematical model to predict dielectric relaxation in glycerol.

Choosing $w=\delta$ in (74) entails $u=\widehat{\chi}$ and verifies that $\widehat{\chi}$ is indeed the impulse response function associated to (74). Choosing $w=w_{0}=\Theta_{-}$in Eq. (74), one obtains $u=\widehat{\chi} * \Theta_{-}$. This solution $u$ of the convolution equation is expected to coincide on $t>0$ with the step relaxation $f$ of Eq. (70) for $h=0$ up to a factor. And that will indeed be verified explicitly further below. For general $h$ the factor $w_{0}$ needs to be replaced with

$$
w_{h}=\Theta_{-}+\frac{h}{1+\tau_{2}^{\alpha} q_{+}^{\alpha}+r_{\mu}^{\alpha} \tau_{2}^{1+\mu(\alpha-1)} \mathrm{q}_{+}^{1+\mu(\alpha-1)}}
$$

and this relates the external forcing $w$ to the inhomogeneity $h$ in Eq. (70). Thus, for $\mu \neq 1$ the right hand side of (70) can not be interpreted as the external force itself but only as a quantity derived from the external force in a non-trivial way.

Because the domain $\mathscr{D}_{L^{1}\left(Q_{-}\right)}^{\prime}$ contains besides $\delta$ and $\Theta_{-}$also the periodic functions $\varpi_{\mathrm{i} \omega}(t)=\exp (\mathrm{i} \omega t), \omega \in \mathbb{R}$ the convolutional formulation Eq. (74) unifies impulse response, step relaxation and periodic excitations. For periodic forcing of the form $w=\varpi_{\mathrm{i} \omega}$ with a single frequency $\omega \in \mathbb{R}$ one obtains the complex admittances

$$
u=\frac{1+\left(\mathrm{i} \tau_{2} \omega\right)^{\alpha}+\mathrm{r}_{\mu}^{\alpha}\left(\mathrm{i} \tau_{2} \omega\right)^{1+\mu(\alpha-1)}}{1+\left(\mathrm{i} \tau_{2} \omega\right)^{\alpha}+\mathrm{i} \tau_{1} \omega} \cdot \varpi_{\mathrm{i} \omega} .
$$

This follows directly from the formulas of the Laplace transforms, because by definition $\mathfrak{L} u(\mathrm{i} \omega)=\mathfrak{F} u(\omega)=\left\langle u, \varpi_{-\mathrm{i} \omega}\right\rangle_{1}=\left(u * \varpi_{\mathrm{i} \omega}\right)(0)$. Equation (76) is just the result expected from multiplier theory.

\subsection{Solutions}

The normalized response function $\widehat{\chi}$, obtained from Eq. (74) for $w=\delta$, can with the help of Eq. (18) be written in the form

$$
\begin{aligned}
\widehat{\chi} & =\frac{\delta+\tau_{2}^{\alpha} \mathrm{q}_{+}^{\alpha}+\mathrm{r}_{\mu}^{\alpha} \tau_{2}^{1+\mu(\alpha-1)} \mathrm{q}_{+}^{1+\mu(\alpha-1)}}{\delta+\tau_{2}^{\alpha} \mathrm{q}_{+}^{\alpha}+\tau_{1} \mathrm{q}_{+}^{1}} \\
& =\mathrm{G}_{\left(-\tau_{2}^{\alpha},-\tau_{1}\right) ;+}^{(\alpha, 1)}+\tau_{2}^{\alpha} \mathrm{G}_{\left(-\tau_{2}^{\alpha},-\tau_{1}\right) ;+}^{(\alpha, 1) ; \alpha}+\mathrm{r}_{\mu}^{\alpha} \tau_{2}^{1+\mu(\alpha-1)} \mathrm{G}_{\left(-\tau_{2}^{\alpha},-\tau_{1}\right) ;+}^{(\alpha, 1) ; 1+\mu(\alpha-1)} \\
& =\frac{1}{\tau_{1}} \mathrm{~F}_{\left(-\tau_{2}^{\alpha} / \tau_{1},-1 / \tau_{1}\right) ;+}^{(1-\alpha, 1) 1}+\frac{\tau_{2}^{\alpha}}{\tau_{1}} \mathrm{~F}_{\left(-\tau_{2}^{\alpha} / \tau_{1},-1 / \tau_{1}\right) ;+}^{(1-\alpha, 1) ; 1-\alpha}+\frac{\mathrm{r}_{\mu}^{\alpha}}{\tau_{1}} \tau_{2}^{1+\mu(\alpha-1)} \mathrm{F}_{\left(-\tau_{2}^{\alpha} / \tau_{1},-1 / \tau_{1}\right) ;+}^{(1-\alpha, 1) ; \mu(1-\alpha)}
\end{aligned}
$$




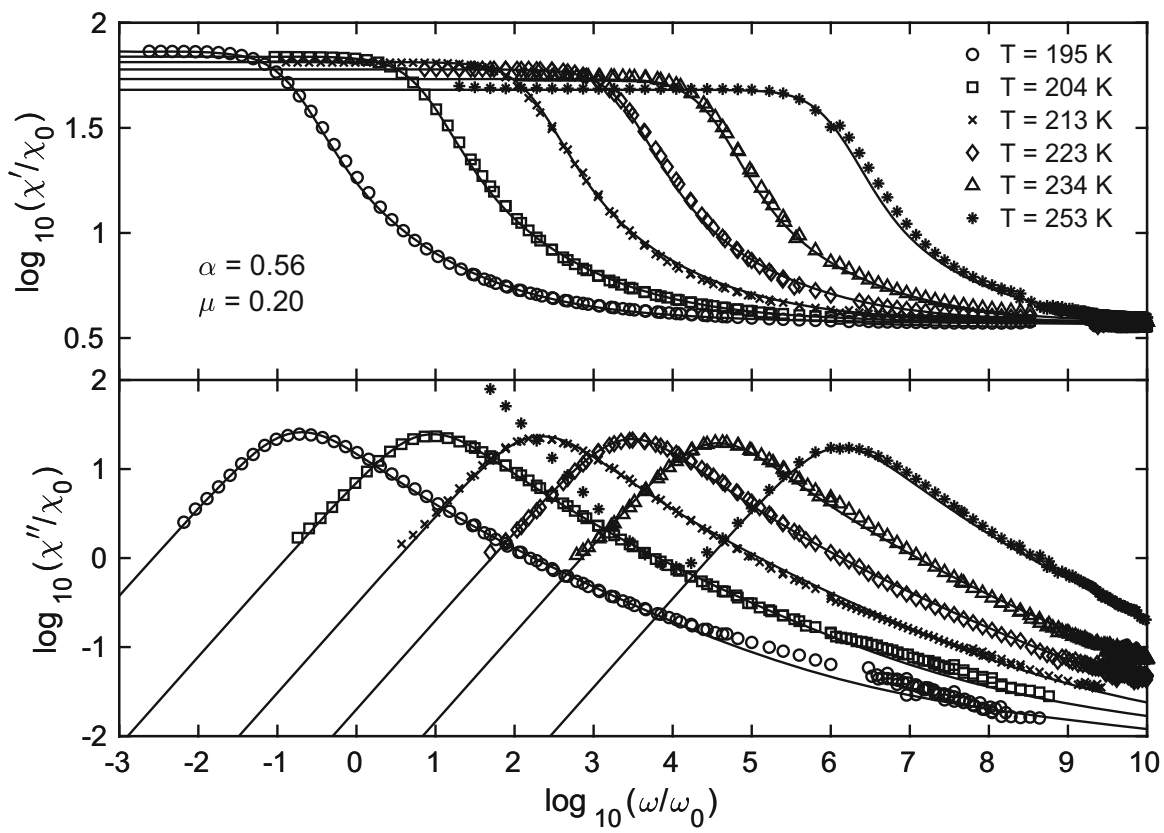

Fig. 1 Fit of dielectric spectra from [25] for glycerol at six different temperatures using Eq. (76) with universal temperature independent dynamical exponent $\alpha=0.56$. The units are $\omega_{0}=2 \pi \mathrm{Hz}$ and $\chi_{0}=$ $10^{7} /\left(4 \pi c^{2}\right) \mathrm{As} / \mathrm{Vm}$

Thus $\widehat{\chi}(t)=0$ for $t \leq 0$ and using Eq. (13b) one finds

$$
\begin{aligned}
\widehat{\chi}(t)= & \frac{1}{\tau_{1}} \mathrm{E}_{(1-\alpha, 1) ; 1}\left(-\frac{\tau_{2}^{\alpha}}{\tau_{1}} t^{1-\alpha},-\frac{t}{\tau_{1}}\right) \\
& +\frac{\tau_{2}^{\alpha}}{\tau_{1}} t^{-\alpha} \mathrm{E}_{(1-\alpha, 1) ; 1-\alpha}\left(-\frac{\tau_{2}^{\alpha}}{\tau_{1}} t^{1-\alpha},-\frac{t}{\tau_{1}}\right) \\
& +\mathrm{r}_{\mu}^{\alpha} \frac{\tau_{2}^{1+\mu(\alpha-1)}}{\tau_{1}} t^{\mu(1-\alpha)-1} \mathrm{E}_{(1-\alpha, 1) ; \mu(1-\alpha)}\left(-\frac{\tau_{2}^{\alpha}}{\tau_{1}} t^{1-\alpha},-\frac{t}{\tau_{1}}\right)
\end{aligned}
$$

for $t>0$.

The subtracted relaxation function $\tilde{f}=f-\chi^{\infty} \Theta_{-}$solving the original problem (70) on $t>0$ can be represented in the form

$$
\begin{aligned}
\tilde{f}= & f-\chi^{\infty} \Theta_{-}=f_{0+} \widehat{\chi} * w_{h}=f_{0+}\left(\frac{\delta+\tau_{2}^{\alpha} \mathrm{q}_{+}^{\alpha}+\mathrm{r}_{\mu}^{\alpha} \tau_{2}^{1+\mu(\alpha-1)} \mathrm{q}_{+}^{1+\mu(\alpha-1)}}{\delta+\tau_{2}^{\alpha} \mathrm{q}_{+}^{\alpha}+\tau_{1} \mathrm{q}_{+}^{1}}\right) \\
& *\left(\Theta_{-}+\frac{h}{\delta+\tau_{2}^{\alpha} \mathrm{q}_{+}^{\alpha}+\mathrm{r}_{\mu}^{\alpha} \tau_{2}^{1+\mu(\alpha-1)} \mathrm{q}_{+}^{1+\mu(\alpha-1)}}\right) .
\end{aligned}
$$


A sufficient assumption on $h$ to make eqs. (70), (74) and (79) well defined is that $h$ is a continuous function with supp $h \subseteq(0,+\infty)$. Because $\mathscr{R}_{+}=\left(\mathscr{R}_{+},+, *\right)$ is an algebra operating on $\mathscr{D}_{L^{1}\left(Q_{-}\right)}^{\prime}$ this expression can be further evaluated. We illustrate the calculations for the homogeneous case $h=0$. One calculates

$$
\begin{aligned}
\frac{\tilde{f}}{f_{0+}} & =1-\widehat{\chi} * \mathrm{p}_{+}^{1}=\Theta_{-}+\Theta_{+}-\mathrm{p}_{+}^{1} *\left(\frac{\delta+\tau_{2}^{\alpha} \mathrm{q}_{+}^{\alpha}+\mathrm{r}_{\mu}^{\alpha} \tau_{2}^{1+\mu(\alpha-1)} \mathrm{q}_{+}^{1+\mu(\alpha-1)}}{\delta+\tau_{2}^{\alpha} \mathrm{q}_{+}^{\alpha}+\tau_{1} \mathrm{q}_{+}^{1}}\right) \\
& =\Theta_{-}+\mathrm{p}_{+}^{1} *\left(\delta-\frac{\delta+\tau_{2}^{\alpha} \mathrm{q}_{+}^{\alpha}+\mathrm{r}_{\mu}^{\alpha} \tau_{2}^{1+\mu(\alpha-1)} \mathrm{q}_{+}^{1+\mu(\alpha-1)}}{\delta+\tau_{2}^{\alpha} \mathrm{q}_{+}^{\alpha}+\tau_{1} \mathrm{q}_{+}^{1}}\right) \\
& =\Theta_{-}+\frac{\tau_{1} \delta}{\delta+\tau_{2}^{\alpha} \mathrm{q}_{+}^{\alpha}+\tau_{1} \mathrm{q}_{+}^{1}}-\frac{\mathrm{r}_{\mu}^{\alpha} \tau_{2}^{1+\mu(\alpha-1)} \mathrm{q}_{+}^{\mu(\alpha-1)}}{\delta+\tau_{2}^{\alpha} \mathrm{q}_{+}^{\alpha}+\tau_{1} \mathrm{q}_{+}^{1}} \\
& =\Theta_{-}+\tau_{1} \mathrm{G}_{\left(-\tau_{2}^{\alpha},-\tau_{1}\right) ;+}^{(\alpha, 1)}-\mathrm{r}_{\mu}^{\alpha} \tau_{2}^{1+\mu(\alpha-1)} \mathrm{G}_{\left(-\tau_{2}^{\alpha},-\tau_{1}\right) ;+}^{(\alpha, 1)} \\
& =\Theta_{-}+\mathrm{F}_{\left(-\tau_{2}^{\alpha} / \tau_{1},-1 / \tau_{1}\right) ;+}^{(1-\alpha, 1) ; 1}-\frac{\mathrm{r}_{\mu}^{\alpha}}{\tau_{1}} \tau_{2}^{1+\mu(\alpha-1)} \mathrm{F}_{\left(-\tau_{2}^{\alpha} / \tau_{1},-1 / \tau_{1}\right) ;+}^{(1-\alpha, 1) ; 1+\mu(1-\alpha)}
\end{aligned}
$$

where eqs. (72), (77) and (18), were used. Using (13b) one finds

$$
\begin{aligned}
\tilde{f}(t)= & f_{0+} \mathrm{E}_{(1-\alpha, 1) ; 1}\left(-\frac{\tau_{2}^{\alpha}}{\tau_{1}} t^{1-\alpha},-\frac{t}{\tau_{1}}\right) \\
& -f_{0+} \frac{\mathrm{r}_{\mu}^{\alpha}}{\tau_{1}} \tau_{2}^{1+\mu(\alpha-1)} t^{\mu(1-\alpha)} \mathrm{E}_{(1-\alpha, 1) ; 1+\mu(1-\alpha)}\left(-\frac{\tau_{2}^{\alpha}}{\tau_{1}} t^{1-\alpha},-\frac{t}{\tau_{1}}\right)
\end{aligned}
$$

for $t>0$ in terms of the binomial Mittag-Leffler function $\mathrm{E}_{\left(\alpha_{1}, \alpha_{2}\right) ; \beta}\left(z_{1}, z_{2}\right)$. In order to verify that $f$ indeed solves (70) the Laplace transform $\mathfrak{L}_{+} f=\mathfrak{L}\left(\Theta_{+} f\right)$ is calculated from Eq. (81) which yields Eqs. (35) and (37) from [10]. This argument works also for non-zero $h$, if $h$ is assumed to be Laplace transformable.

\subsection{Application to glycerol}

In this section the convolution Eq. (74) is applied to predict broadband dielectric spectra of glycerol over 13 decades in frequency and temperatures between 193 and $253 \mathrm{~K}$. The experimental data are from [25]. Figure 1 shows the result of fitting Eq. (76) to the experimental data. In all fits the order $\alpha=0.56$ of the fractional derivative is fixed and independent of temperature. It plays the role of a universal dynamical critical exponent. The type $\mu=0.2$ and the parameter $\mathrm{r}_{\mu}^{\alpha}$ are also temperature independent. Moreover, the time scale ratio $\tau_{1} / \tau_{2}$ is only weakly temperature dependent varying monotonically between $\tau_{1} / \tau_{2} \approx 4.57$ for $T=195 \mathrm{~K}$ and $\tau_{1} / \tau_{2} \approx 6.45$ for $T=253 \mathrm{~K}$. This leaves a single relaxation time, e.g. $\tau_{1}$, as the only temperature dependent fit parameter. It determines the frequency of the main peak in the imaginary part. Note that previously there did not exist a fit function for the excess wing [25] and its physical origin was unknown. Remarkably, the composite fractional relaxation model (70) and 
its convolutional interpretation (74) predict asymmetric relaxation peaks plus excess wings with a single temperature independent scaling exponent.

Funding Open Access funding enabled and organized by Projekt DEAL.

Data Availability Not applicable.

\section{Declarations}

Conflict of interest Not applicable.

Open Access This article is licensed under a Creative Commons Attribution 4.0 International License, which permits use, sharing, adaptation, distribution and reproduction in any medium or format, as long as you give appropriate credit to the original author(s) and the source, provide a link to the Creative Commons licence, and indicate if changes were made. The images or other third party material in this article are included in the article's Creative Commons licence, unless indicated otherwise in a credit line to the material. If material is not included in the article's Creative Commons licence and your intended use is not permitted by statutory regulation or exceeds the permitted use, you will need to obtain permission directly from the copyright holder. To view a copy of this licence, visit http://creativecommons.org/licenses/by/4.0/.

\section{References}

1. Atanackovic, T., Oparnica, L., Pilipovic, S.: Semilinear ordinary differential equation coupled with distributed order fractional differential equation. Nonlinear Anal. 72, 4101-4114 (2010)

2. Balakrishnan, A.: Fractional powers of closed operators and the semigroups generated by them. Pacific J. Math. 10, 419-437 (1960)

3. Cresson, J.: Fractional embedding of differential operators and Lagrangian systems. J. Math. Phys. 48, 033504 (2007)

4. Dierolf, P., Voigt, J.: Convolution and S'-convolution of distributions. Collect. Math. 29, 185-196 (1978)

5. Dipierro, S., Palatucci, G., Valdinoci, E.: Dislocation Dynamics in Crystals: A Macroscopic Theory in a Fractional Laplace Setting. Commun. Math. Phys. 333, 1061-1105 (2015)

6. Erdelyi, A.: Fractional integrals of generalized functions. In: Ross, B. (ed.) Fractional Calculus and its Applications. Lecture Notes in Mathematics, vol. 457, pp. 151-170. Springer Verlag, Berlin (1975)

7. Gelfand, I., Shilov, G.: Generalized Functions, vol. I. Academic Press, New York (1964)

8. Godement, R.: Analysis IV: Integration and Spectral Theory. Springer, Berlin (2015)

9. Hilfer, R.: Applications of Fractional Calculus in Physics. World Scientific Publ. Co., Singapore (2000)

10. Hilfer, R.: Experimental evidence for fractional time evolution in glass forming materials. Chem. Phys. 284, 399 (2002)

11. Hilfer, R.: Excess wing physics and nearly constant loss in glasses. J. Stat. Mech. Theory Exp. 2019, 104007 (2019)

12. Hilfer, R.: Mathematical and physical interpretations of fractional derivatives and integrals. In: Kochubei, A., Luchko, Y. (eds.) Handbook of Fractional Calculus with Applications: Basic Theory, vol. 1, pp. 47-86. Walter de Gruyter GmbH, Berlin (2019)

13. Hilfer, R., Luchko, Y., Tomovski, Z.: Operational method for the solution of fractional differential equations with generalized Riemann-Liouville fractional derivatives. Fract. Calc. Appl. Anal. 12, 299 (2009)

14. Horvath, J.: Topological Vector Spaces and Distributions. Addison-Wesley, Reading (1966)

15. Hövel, H., Westphal, U.: Fractional powers of closed operators. Studia Math. 42, 177-194 (1972)

16. Kamocki, R.: A new representation formula for the Hilfer fractional derivative and its application. J. Comput. Appl. Math. 308, 39-45 (2016)

17. Kato, T.: Note on fractional powers of linear operators. Proc. Japan Acad. 36, 94-96 (1960)

18. Kleiner, T., Hilfer, R.: Weyl integrals on weighted spaces. Fract. Calc. Appl. Anal. 22, 1225-1248 (2019) 
19. Kleiner, T., Hilfer, R.: Convolution operators on weighted spaces of continuous functions and supremal convolution. Annali di Matematica 199, 1547-1569 (2020)

20. Komatsu, H.: Fractional powers of operators. Pac. J. Math. 19, 285-346 (1966)

21. La Nave, G., Limtragool, K., Phillips, P.: Colloquium: Fractional electromagnetism in quantum matter and high-energy physics. Rev. Mod. Phys. 91, 021003-1 (2019)

22. Lamb, W.: A distributional theory of fractional calculus. Proc. R. Soc. Edinb. 99A, 347-357 (1985)

23. Lanford, O., Robinson, D.: Fractional powers of generators of equicontinuous semigroups and fractional derivatives. J. Aust. Math. Soc. (A) 46, 473-504 (1989)

24. Luchko, Y., Gorenflo, R.: An Operational Method for Solving Fractional Differential Equations with the Caputo Derivatives. Acta Math. Vietnam. 24, 207-233 (1999)

25. Lunkenheimer, P., Loidl, A.: Dielectric spectroscopy of glass-forming materials: $\alpha$-relaxation and excess wing. Chem. Phys. 284, 205-219 (2002)

26. Marchaud, A.: Sur les derivees et sur les differences des fonctions de variables reelles. J. Math. Pures Appl. 6, 337-425 (1927)

27. Martinez Carracedo, C., Sanz Alix, M.: The Theory of Fractional Powers of Operators. Elsevier, Amsterdam (2001)

28. McBride, A.: Fractional Calculus and Integral Transform of Generalized Functions. Pitman Publishing Ltd, San Francisco (1979)

29. McIntosh, A.: Operators which have an $H_{\infty}$-calculus. In: Jefferies, B., et al. (eds.) Miniconference on Operator Theory and Partial Differential Equations, pp. 210-231. Australian National University, Canberra (1986)

30. Mikusinski, J.: Operational Calculus. PWN, Warszaw (1959)

31. Miller, K.: The Weyl fractional calculus. In: Ross, B. (ed.) Fractional Calculus and its Applications. Lecture Notes in Mathematics, vol. 457, pp. 80-89. Springer Verlag, Berlin (1975)

32. Miller, K., Ross, B.: An Introduction to the Fractional Calculus and Fractional Differential Equations. Wiley, New York (1993)

33. Morita, T., Sato, K.: Neumann-series solution of fractional differential equation. Interdiscip. Inf. Sci. 16, 127-137 (2010)

34. Ortner, N.: On some contributions of John Horvath to the theory of distributions. J. Math. Anal. Appl. 297, 353-383 (2004)

35. Ortner, N., Wagner, P.: Distribution-Valued Analytic Functions - Theory and Applications. Tredition GmbH, Hamburg (2013)

36. Ortner, N., Wagner, P.: Fundamental Solutions of Linear Partial Differential Operators. Springer, Berlin (2015)

37. Pietsch, A.: Nuclear Locally Convex Spaces. Springer, Berlin (1972)

38. Prüss, J., Spohr, H.: On operators with bounded imaginary powers in banach spaces. Math. Z. 203, 429-452 (1990)

39. Samko, S., Kilbas, A., Marichev, O.: Fractional Integrals and Derivatives. Gordon and Breach, Berlin (1993)

40. Saxena, R., Garra, R., Orsingher, E.: Analytical solution of space-time fractional telegraph-type equations involving Hilfer and Hadamard derivatives. Integral Transf. Special Funct. 27, 30-42 (2016)

41. Schiavone, S., Lamb, W.: A fractional power approach to fractional calculus. J. Math. Anal. Appl. 149, 377-401 (1990)

42. Schwartz, L.: Theorie des Distributions. Hermann, Paris (1950)

43. Schwartz, L.: Definition integrale de la convolution de deux distributions. Seminaire Schwartz 1, 1-7 (1954)

44. Uiterdijk, M.: A functional calculus for analytic generators of $C_{0}$-Groups. Integr. Eqn. Oper. Theory 36, 340-369 (2000)

45. Westphal, U.: Ein Kalkül für gebrochene Potenzen infinitesimaler Erzeuger von Halbgruppen und Gruppen von Operatoren. Teil I: Halbgruppenerzeuger. Compositio Math. 22, 67-103 (1970)

46. Westphal, U.: Fractional powers of infinitesimal generators of semigroups. In: Hilfer, R. (ed.) Applications of Fractional Calculus in Physics, pp. 131-170. World Scientific, Singapore (2000)

47. Yosida, K.: Fractional powers of infinitesimal generators and the analyticity of the semi-groups generated by them. Proc. Japan Acad. 36, 86-89 (1960)

48. Yosida, K.: Functional Analysis. Springer, Berlin (1965)

49. Zavada, P.: Operator of fractional derivative in the complex plane. Commun. Math. Phys. 192, 261-285 (1998) 
50. Zemanian, A.: Distribution Theory and Transform Analysis. McGraw-Hill, New York (1965)

Publisher's Note Springer Nature remains neutral with regard to jurisdictional claims in published maps and institutional affiliations. 